\title{
The Dictyostelium discoideum E3 ubiquitin ligase TrafE coordinates endolysosomal damage response and cell-autonomous immunity to Mycobacterium marinum.
}

\author{
Lyudmil Raykov, Manon Mottet, Jahn Nitschke, Thierry Soldati*
}

Départment de Biochimie, Faculté des Sciences, Université de Genève, Sciences II, 30 quai Ernest Ansermet, CH-1211Genève-4, Switzerland.

*Correspondence to: Thierry Soldati, thierry.soldati@unige.ch, Tel: +41 223796496

\begin{abstract}
Cells are perpetually challenged by pathogens, protein aggregates or chemicals, that induce plasma membrane or endolysosomal compartments damage. Endolysosomal perforations are recognised as severe stress, however the mechanisms of the cellular response that ensure quality control, repair and endolysosomal homeostasis are just beginning to be unravelled. The endosomal sorting complex required for transport (ESCRT) and the autophagy machinery are recruited to damaged membranes to either repair or to remove membrane remnants. Crucial element of the endolysosomal damage response (ELDR) are factors that sense damage, paralleled by extensive tagging of the damaged organelles
\end{abstract}

\section{INTRODUCTION}

After its internalisation by professional phagocytes, the first strategy of Mycobacterium tuberculosis and its close relative Mycobacterium marinum to escape the bactericidal phagosome is to impede its maturation and tailor the MycobacteriumContaining Vacuole (MCV) as a permissive niche allowing the proliferation of the pathogen (Soldati and Neyrolles, 2012). From the very beginning, bacteria progressively gain access to the cytosol through the action of the ESX-1 secretion system, via the secretion of the membranolytic effector ESAT-6 and the mycobacterial branched apolar lipids phthiocerol dimycocerosates (PDIMs) (Pym et al., 2002; Lerner et al., 2018; Quigley et al., 2017; Augenstreich et al., 2019; Lienard et al., 2020; Osman et al., 2020). The ESX-1 with signals, such as ubiquitin, required for the recruitment of ELDR components. Unattended membrane damage leads to leakage of harmful components including protons and reactive oxygen species that cause cell death. To explore ELDR key factors responsible for detection and marking of damaged compartments we use the professional phagocyte Dictyostelium discoideum. We found an evolutionary conserved E3-ligase TrafE that is robustly recruited to intracellular compartments disrupted after infection with Mycobacterium marinum or after sterile damage caused by chemical components. Importantly, we show that the absence of TrafE severely compromises the xenophagy restriction of bacteria as well as autophagy-mediated and ESCRT-mediated ELDR, resulting in early cell death.

secretion system plays a crucial role for $M$. marinum virulence and intracellular growth, and is primarily encoded by the genes within the "region of difference 1" (RD1) locus. Lessons from professional phagocytes such as the mammalian macrophages and the social amoeba Dictyostelium discoideum show that $M$. marinum mutants with non-functional ESX-1 inflict significantly less MCV membrane damage and fail to become exposed to or escape to the cytosol, hampering their detection by the host cell-autonomous defence machineries (Cardenal-Muñoz et al., 2017; Gao et al., 2004). Once in contact with the cytosol, the wild-type (WT) bacteria within damaged MCVs are detected and tagged with signals which trigger ELDR as well as selective anti-bacterial autophagy (López-Jiménez et al., 2019). To complete the infection cycle, the pathogen must 
reach the cytosol and be released by host-cell lysis, exocytosis or ejection in order to disseminate to adjacent bystander cells (Hagedorn et al., 2009; Queval et al., 2017; Cardenal-Muñoz et al., 2017; López-Jiménez et al., 2019).

Eukaryotic cells target intracellular invaders towards the pathogen-specific autophagy pathway termed xenophagy (hereafter referred to as autophagy), a process which plays a fundamental role in cell-autonomous immunity (Sharma et al., 2018; Deretic et al., 2013). Upstream, autophagy requires the detection of the pathogen and its recruitment to the phagophore - a double membrane cup-like structure. Autophagy depends on the coordinated action of about 15 autophagyrelated genes (ATG) and is initiated by the recruitment of the core-autophagy factors Atg1 (ULK1 in mammals) kinase complex and Atg9 (ATG9 in mammals) to the nascent phagophore. Its closure around the cargo depends on the atg16-atg12-atg5 E3-like complex, and the lipidation of Atg8 (LC3 in mammals), which marks the phagophore (Mesquita et al., 2017). The resulting autophagosome fuses with lysosomes allowing the degradation of their contents. The selectivity is achieved by the autophagy cargo receptors, which bring together the cargo and the autophagy machinery. Autophagy adaptors such as p62 (SQSTM1 in mammals) link ubiquitin-tagged bacteria and host components with Atg8 family proteins on the phagophore membranes, thereby enforcing proximity between the pathogen and the autophagosome (Boyle and Randow, 2013). We have shown previously that, upon infection, $M$. marinum does colocalize with ubiquitin (Ub) and the autophagy markers such as Atg8 and p62, and that autophagy deficiency results in drastic increase in bacteria proliferation, and that failure to repair or recycle damaged MCVs results in increased cell death (Cardenal-Muñoz et al., 2017; Eriksson et al., 2020; Wang et al., 2018). Autophagy is also involved in ELDR, and beside the elimination of extensively damaged compartments by a specific macroautophagy termed lysophagy, it cooperates with ESCRT to repair sterile- or pathogen-induced membrane damage (Skowyra et al., 2018; López-Jiménez et al., 2019; Papadopoulos et al., 2020). The ESCRTIII complex functions in membrane deformation away from the cytosol and plays a critical role in various membrane remodelling processes, including phagophore sealing, autophagosome-lysosome fusion, plasma membrane and lysosome membrane repair (Vietri et al., 2020; Schuck, 2020). One thoroughly studied ESCRT function is the coupling of cargo sorting and intralumenal vesicles formation required for degradation of receptors.

The endolysosomal and MCV membrane ruptures generate morphological and topological landscape for the detection machineries which deposit "repair-me" and "eat-me" signals (Boyle and Randow, 2013; Koerver et al., 2019; Papadopoulos et al., 2020). Ubiquitination is a well-studied, highly conserved and versatile post-translational modification used as a signalling platform in diverse catabolic processes and playing a role during infection with various intracellular bacteria. In mammalian cells, the RING domain E3 ligases LRSAM1 and TRAF6 have been shown to promote ubiquitination of vacuoles containing Salmonella Typhimurium and Chlamydia trachomatis bacteria, and the protozoan parasite Toxoplasma gondii (Coers and Haldar, 2015; Huett et al., 2012), and NEDD4 (Pei et al., 2017), Parkin (Manzanillo et al., 2013), Smurf1 (Franco et al., 2017) to mediate ubiquitination of mycobacteria and host proteins during infection. In addition, the mammalian cytosolic lectins, galectins, recognize damage by binding exposed glycans on the luminal membrane leaflets and components of bacteria cell wall, thereby serving as "eat-me" tags for autophagy. Moreover, complexes such as TRIM16Galectin3 also mediate autophagy removal of damaged phagosomes and lysosomes during pathogen invasion (Chauhan et al., 2016; Randow and Youle, 2014). The human tumour necrosis factor receptor-associated factors (TRAF1-7) function in a number of biological processes such as innate and adaptive immunity and cell death as E3 ubiquitin ligases and scaffold proteins (Xie, 2013). TRAFs are composed of several domains: the N-terminal domain containing a RING motif (except TRAF1), a series of $\mathrm{Zn}$-finger motifs that connect the $\mathrm{N}$ - and $\mathrm{C}$-terminal regions and the C-terminal TRAF domain (except for TRAF7) subdivided in TRAF-N coiled-coil domain and TRAF-C domain comprised of seven to eight 
antiparallel $\beta$-strands (Bradley and Pober, 2001; Park, 2018; Joazeiro and Weissman, 2000). The RING domain and the Zn-finger motifs of TRAF6 are required for the E3 ligase activity and together with the dimeric E2conjugating enzyme complex Ubc13-UeV1A, TRAF6 catalyses the generation of poly-Ub chains linked via the lysin K63 of $\mathrm{Ub}$, in a homo- or hetero-oligomerisation-dependent manner with other TRAF members (Middleton et al., 2017). The scaffolding function of TRAF proteins is conferred by the TRAF-N domain responsible for homo- and heterooligomerisation, which is also crucial for the E3 ligase activity, and the TRAF-C domain allows interaction with upstream receptors and adaptors (Yin et al., 2010). In recent years, the selective autophagy and maintenance of the endolysosomal homeostasis have been actively studied and linked to many aspects of human health. Endomembrane ruptures on one hand permit the pathogen to gain access to the cytosol, but on the other hand to be detected. Then, together with the vacuole membrane remnants, bacteria are targeted to the autophagy machinery. Defects in endomembrane repair and lysophagy correlate with weakened cellular defences against pathogens, ageing, cancer and neurodegeneration (Papadopoulos and Meyer, 2017). Even though the role of "eat-me" signals in autophagy is well understood, little is known about their implication in detection and repair of pathogen-induced or sterile membrane damage. Furthermore, how pathogen and membrane damages are detected, leading to the deposition of such "eat-me" signals, remains unknown.

Using the social amoeba Dictyostelium discoideum - M. marinum model system for host-pathogen interactions, we have unravelled a novel function of a TRAF-like family protein RING domain-containing E3 ligase. Among a dozen candidates explored, we found that TrafE is an evolutionary conserved E3-ligase, proximal to the bacteria detection machinery, which plays a key role in the preservation of endomembrane integrity during infection with $M$. marinum and upon sterile damage. Importantly, we provide evidence that in the absence of TrafE, the autophagy restriction of bacteria as well as the autophagy-mediated and ESCRT-mediated ELDR are severely compromised, leading to precocious cell death.

\section{RESULTS}

\section{The E3-ligase TrafE is overexpressed early- on after infection and is recruited to MCVs}

Using the sequence of the human TRAF6 as a reference we searched and identified bioinformatically a number of $D$. discoideum TRAF-like proteins, five of which, namely TrafA, TrafB, TrafC, TrafD and TrafE harbour all of the stereotypical TRAF protein family domains and are expressed during the vegetative cell cycle (Dunn et al., 2018; Stajdohar et al., 2017). In parallel, RNAsequencing (RNA-seq) transcriptomic comparison between control and M. marinuminfected $D$. discoideum cells showed that out of the aforementioned candidates only trafE is overexpressed early after infection (S1 Fig) (Hanna et al., 2019) and enriched at the early $\mathrm{MCV}$, as revealed by proteomics (Guého et al., 2019).

To validate a possible role in infection of the aforementioned TRAF-like proteins, we first sought to identify candidates that display remarkable relocalization during infection. To do so, we generated stably transformed cell lines ectopically expressing TrafA, TrafB, TrafC, TrafD and TrafE candidate proteins, Nor C-terminally fused to GFP, under a constitutive promoter. We monitored the subcellular localization of these GFP fusions by live confocal microscopy over a period of 24 hours in the context of an infection with $M$. marinum, compared to a mock-infected control. As early as 1 hour post infection (hpi), GFPTrafE showed robust recruitment to $\mathrm{MCV}$, sustained over the infection time course, predominantly in the area of $M$. marinum bacteria poles (Fig 1A, 1B and 1C). GFP-TrafA and GFP-TrafB remained solely cytosolic or nuclear, respectively, in control and infection conditions at all time points (S2A and S2B Fig). We confirmed in silico that TrafB harbours a nuclear localization signal (NLS) similarly to human TRAF4, as predicted by both NLS Mapper and NLStradamus (Nguyen Ba et al., 2009; Kosugi et al., 2009). Interestingly, despite TrafC and TrafD being 98\% similar in terms of amino acid sequence, they displayed different behaviour upon infection. GFP-TrafC like GFP-TrafE was recruited to MCV, which was not the case for GFP-TrafD (S3A and S3B Fig). 
Real-Time quantitative-PCR (RT q-PCR) carried out to monitor the transcriptional profile in control and infected cells confirmed up to 4fold upregulation of trafE already at $1 \mathrm{hpi}$ (Fig 1D), consistent with the RNA-seq data (Hanna et al., 2019). We also evaluated a possible overexpression phenotype of TrafC, TrafD and TrafE during infection using an intracellular growth assay during infection with bioluminescent M. marinum (M. marinum-lux). In this assay the bioluminescence intensity is measured in relative light units (RLU) as a readout for bacterial growth (Sattler et al., 2007). Whilst the intracellular growth of $M$. marinum WT remained unaffected in cells overexpressing TrafC or TrafD, this growth was significantly hampered when TrafE was overexpressed (S4A Fig, Fig 1E). These results persuaded us to explore further the role of TrafE in infection as potentially the principal E3 ligase candidate.

In order to assess potential artefacts of overexpression, a TrafE-GFP chromosomal knock-in (TrafE-GFP KI) was generated and tested functionally by monitoring intracellular growth of $M$. marinum-lux. Importantly, bacteria growth in the TrafE-GFP KI strain was comparable to that in WT cells (S4B Fig), indicating that C-terminal GFP fusion of TrafE does not interfere with its function. In addition, the endogenous TrafE-GFP colocalized with MCVs already 1.5 hours after infection (Fig 1F) like the ectopically expressed GFP-TrafE (Fig 1A). Thus, our data suggests that TrafE is involved in the early MCV membrane damage detection and MCV or M. marinum ubiquitination, necessary for repair or degradation. We conclude that TrafE is a conserved E3-ligase upregulated and recruited to the $M$. marinum compartment upon infection and likely involved in $D$. discoideum cellautonomous immunity.

\section{Loss of TrafE promotes $M$. marinum early release and replication, and is highly toxic for host cells}

To explore further whether TrafE is either involved in the host defence response or is exploited by bacteria to favour their proliferation, we generated cell lines in which the trafE coding sequence (CDS) was ablated (trafE KO), and strains overexpressing TrafE. We observed that the load of M. marinum-lux was increased in trafE $\mathrm{KO}$ cells and, most interestingly, was comparable and phenocopied the growth of the pathogen in the autophagyimpaired atgl KO mutant cells (Fig 2A).

Previous studies indicated that in the absence of autophagy (e.g. atgl KO cells) or ESCRT-IIImediated membrane damage repair (e.g., tsg101 KO cells), bacteria escape early to the cytosol (Cardenal-Muñoz et al., 2017; LópezJiménez et al., 2019). As a reporter for $M$. marinum escape to the cytosol, we monitored Plin, the $D$. discoideum homolog of the mammalian perilipins known to bind the hydrophobic cell wall of mycobacteria exposed to the cytosol (Barisch et al., 2015b). The proportion of mCherry-Plin-positive bacteria indicated that in trafE $\mathrm{KO}$ host cells $M$. marinum was exposed to the cytosol significantly earlier, compared to WT cells, but similar to atgl KO cells (Fig 2B). It has been shown that in the atgl $\mathrm{KO}$ and tsg101 KO mutant cells $M$. marinum escapes earlier from the MCV to the cytosol due to a lack of both autophagic and ESCRT-III MCV membrane repair (Cardenal-Muñoz et al., 2017; LópezJiménez et al., 2019). However, in the context of these mutations $M$. marinum has distinct fates. In atgl $\mathrm{KO}$ cells, bacteria accumulate ubiquitin but proliferate significantly due to a lack of functional autophagy. In tsg101 KO background $M$. marinum is ubiquitinated and recruited to the autophagy machinery, leading to restriction of bacterial growth. Interestingly, in trafE $\mathrm{KO}$ host cells M. marinum escapes earlier and displays high proliferation, suggesting that both MCV damage repair and xenophagy restriction of bacteria are impaired. Furthermore, we infected WT and trafE KO cells with mCherry-expressing bacteria and monitored the infection by high-content (HC) confocal microscopy. Surprisingly, after $30 \mathrm{hpi}$ we observed a drastic decrease in the number of infected trafE $\mathrm{KO}$ cells compared to infected WT cells (Fig 2C). This was paralleled by a high number of extracellular $M$. marinum that had likely been released by lysis from host trafE mutant cells near 30 hpi (Fig 2D). In conclusion, we hypothesized that $M$. marinum escapes earlier to the cytosol of trafE KO cells and as a likely consequence, kills the host faster.

To validate this hypothesis, we employed a microfluidic device in which single infected cells are trapped in the InfectChip (Delincé et al., 2016). We confirmed that M. marinum is 
highly toxic for trafE KO cells, a phenotype almost fully complemented by GFP-TrafE overexpression (Fig. 2E). Surprisingly, the survival probability of infected autophagydeficient atgl KO host cells was not significantly affected under these conditions and time-frame (Fig 2E), even though $M$. marinum intracellular growth is drastically increased in these mutants compared to WT (Cardenal-Muñoz et al., 2017). Altogether, these results reveal that absence of Atg1 or TrafE facilitates $M$. marinum early escape from the MCVs to the cytosol, and strongly affects its targeting to the xenophagy machinery. As a consequence, the pathogen rapidly proliferates, suggesting that both Atg1 and TrafE work in the same pathway. Nevertheless, M. marinum is more toxic to $D$. discoideum in absence of TrafE than of Atg1, indicating that TrafE is more broadly involved in cell survival mechanisms.

\section{TrafE action is triggered by $M$. marinum- induced MCV membrane damage}

Taking into account that MCV membrane disruption prompts the recruitment of both the ESCRT and the autophagy machineries, we hypothesized that TrafE colocalization with $M$. marinum depends on membrane damage. Both, $M$. tuberculosis and $M$. marinum share the genomic locus Region of Difference 1 (RD1) encoding the ESX-I (type VII) secretion system required for the secretion of the membranolytic virulence factor ESAT-6 (Hagedorn et al., 2009). As previously shown (Cardenal-Muñoz et al., 2017), in $D$. discoideum cells infected with M. marinum $\triangle \mathrm{RD} 1$, which induces less MCV damages, bacteria and their compartment ubiquitination is significantly decreased. In addition, nearly $80 \%$ of GFP-TrafE recruited to MCVs is in the vicinity of bacteria poles (Fig 1C), where ESX-1 is localized and active in both M. tuberculosis and M. marinum (Carlsson et al., 2009). Interestingly, upon infection with M. marinum $\triangle \mathrm{RD} 1$, TrafE-GFP recruitment to MCV was severely reduced (Fig 3A and 3B). Previously, we showed that both intracellular growth of WT $M$. marinum as well as autophagosome formation depend on a functional ESX-1 system. Consequently, the bacteria load of $M$. marinum $\triangle \mathrm{RD} 1$ was significantly and similarly reduced in both WT and atgl KO cells (Cardenal-Muñoz et al., 2017). Therefore, we measured the intracellular growth of WT and M. marinum $\triangle \mathrm{RD} 1$ bacteria.
Lack of the ESX-1 system drastically reduced bacterial proliferation in both WT and trafE KO cells (Fig 3C), indicating that the growth advantage of WT M. marinum in trafE KO cells is strictly dependent on MCV damage and escape to the cytosol. In addition, the survival probability of both trafE KO and atgl KO host cells was not affected during infection with the M. marinum $\Delta \mathrm{RD} 1$ bacteria (Fig 3D). In conclusion, we propose that membrane damage is required for TrafE recruitment to MCVs and subsequent restriction of bacteria proliferation by xenophagy.

\section{K63-ubiquitination levels are decreased in the absence of TrafE}

A hallmark of damaged MCVs is ubiquitination that plays an essential role in pathways such as cell survival (e.g. autophagy), intracellular pathogen clearance (e.g. xenophagy) and cell death (e.g. apoptosis, necrosis) (CardenalMuñoz et al., 2017; Papadopoulos and Meyer, 2017; Yoshida et al., 2017; López-Jiménez et al., 2019; Gómez-Díaz and Ikeda, 2019; Papadopoulos et al., 2020). We carried out immunofluorescence assays using antibodies specific to K63-linkage polyubiquitin and determined that GFP-TrafE colocalized with K63-ubiquitinylated MCVs (Fig 4A). Next, we compared the total levels of polyubiquitinpositive MCVs or K63-linked polyubiquitinylated MCVs in infected WT or trafE KO cells, using a global antipolyubiquitin antibody or the specific K63linkage polyubiquitin antibody. Interestingly, at $6 \mathrm{hpi}$ the global levels of polyubiquitination between trafE $\mathrm{KO}$ and WT cells remained comparable, while the fraction of K63-linkage positive MCVs was drastically reduced in trafE KO cells (Fig 4A and 4B). These results suggest that TrafE, as part of the ELDR, functions as a E3 ligase responsible for K63-linked polyubiquitination of MCVs and bacteria upon membrane damage.

\section{TrafE recruitment to bacteria is mediated by its RING domain}

It has been previously reported, that TRAF E3ligase activity depends on the RING domain that mediates the interaction between the E2conjugating enzymes and their substrates, and TRAF-N and RING domains-dependent homoor hetero-oligomerizations with other members of the TRAF family (Das et al., 2021). Therefore, in order to dissect the functional 
importance of the RING and the TRAF-N regions during $M$. marinum infection, we generated truncated versions of TrafE that were expressed in the trafE KO background. Absence of the N-terminal RING domain resulted in complete loss of TrafE recruitment to MCVs throughout the infection time-course, in contrast to the truncated TRAF-N domain version or the full-length protein (Fig 5A). Despite that, functional assays using $M$. marinum-lux indicated that both deleted protein domains fail to complement the trafE KO (Fig $5 \mathrm{~B})$. We reason that the absence of RING domain phenocopies the truncation of the TRAF-N region because both are required for the TrafE E3 ligase activity. Additionally, the RING domain determines substrate recognition (Jackson et al., 2000) and binding to E2conjugating enzymes, which might explain why in its absence, TrafE is not recruited to damaged MCVs.

\section{Sterile damage triggers TrafE recruitment on endolysosomes}

Late endosomes and lysosomes are involved in the degradation of molecules endocytosed from the extracellular space or captured by the autophagy machinery in the cytosol (Huotari and Helenius, 2011). In D. discoideum, autophagy and ESCRT cooperate to promote membrane repair, however, extensively damaged endolysosomes are targeted to the autophagy machinery for removal (LópezJiménez et al., 2019). To address whether TrafE recruitment to bacteria is specific to infectioninduced MCV damage or represents a general reaction to membrane damage, we employed the esterified dipeptide named L-leucyl-Lleucine methyl ester (LLOMe) which perforates endolysosomes, making them permeable to protons and small molecules of less than 10 $\mathrm{kDa}$, resulting in endolysosomal $\mathrm{pH}$ increase and leading to apoptosis at concentrations above $4 \mathrm{mM}$ in HeLa cells (Repnik et al., 2017). Surprisingly, minutes after treatment with 4.5 $\mathrm{mM}$ LLOMe, the homogenous cytosolic distribution of GFP-TrafE changed dramatically to dispersed puncta and rings (Fig 6A, S1 Movie). The cytosolic GFP-TrafE $\triangle$ TRAF-N was slowly recruited on the LLOMe-damaged compartments, but the cytosolic pattern of GFP-TrafE $\triangle$ RING remained unchanged until the end of the monitoring period (Fig 6B and 6C). A large fraction of TrafE was clearly recruited to Alexa
Fluor 647-conjugated $10 \mathrm{kDa}$ dextran-loaded endolysosomes (Fig 6D, S2 Movie). Our data suggest that the TrafE RING domain is essential for its response to membrane damage and importantly, considering the swiftness of TrafE reaction to LLOMe-induced damages, TrafE is likely an early sensor of damage and an upstream effector of the ELDR.

\section{TrafE is essential for the maintenance of endolysosomal integrity}

Previous results indicated that defects in the ESCRT and autophagy machineries, both known to contribute to membrane repair, lead to increased proton leakage upon LLOMeinduced sterile damage. To monitor how LLOMe affects the endolysosomal integrity and $\mathrm{pH}$ in the context of trafE $\mathrm{KO}$, we used lysosensor DND-189 which is a fluorescent $\mathrm{pH}$ tracer that accumulates in acidic compartments, where protonation relieves quenching. The addition of $4.5 \mathrm{mM}$ LLOMe was followed by a decrease of lysosensor fluorescence intensity in both WT and trafE KO cells, indicating proton leakage and $\mathrm{pH}$ increase within the endolysosomal compartments (Fig 7A). In WT cells, the lysosensor intensity first decreased but was restored to the initial level (Fig 7A and 7B), indicating that WT cells completed recovery in the course of the 25 minutes of LLOMe treatment. In contrast, the lysosensor fluorescence intensity remained low in the trafE mutant cells (Fig 7A and 7B), indicating a more pronounced and irreversible proton leakage. The results show that cells can fully repair damage to endolysosomes and restore their $\mathrm{pH}$, while absence of TrafE has a strong negative impact on the integrity and the repair of damaged compartments.

\section{TrafE is recruited to endolysosomes after hypertonic shock}

LLOMe-induced membrane damage reduces membrane tension similarly to hyperosmotic conditions (Mercier et al., 2020). Indeed, water expulsion from the cytosol and from the endolysosomal compartments results in membrane tension decrease that is sufficient to trigger the recruitment of Alix and the ESCRTIII component CHMP4B and subsequent formation of intraluminal vesicles (ILVs). To reveal possible mechanisms of TrafE recruitment to endolysosomes, we asked whether a membrane tension decrease might be sufficient to trigger recruitment of TrafE to 
endo-membranes. For this, we monitored $D$. discoideum cells expressing GFP-TrafE under hypertonic conditions. Strikingly, cytosolic TrafE relocated to endolysosomal compartments seconds after the addition of sorbitol. In addition, when cells adapted and regained their initial shape and motility, TrafE regained a cytosolic distribution (Figure 8A). The $\mathrm{pH}$-tracer lysosensor was used again to address whether the hyperosmotic shock induced membrane perforations. Addition of high $5 \mathrm{mM}$ LLOMe concentration resulted in a decrease of lysosensor fluorescence intensity indicative of proton leakage from damaged endolysosomes. In contrast, under hypertonic conditions, the lysosensor fluorescence intensity remained similar, indicating that the membrane integrity of acidic compartment was uncompromised (Fig 8B and 8C). Our data suggest that a decrease of membrane tension alone is enough to trigger TrafE recruitment to endolysosomal membranes.

\section{Absence of TrafE confers acute susceptibility to sterile damage}

In the absence of TrafE, the probability of survival of M. marinum-infected D. discoideum cells was drastically decreased compared to infected WT cells (Fig 2A). In addition, recent studies showed that disruption of lysosomal membrane leads to increase of cytosol acidity and necrosis or lysosome-dependent cell death (Wang et al., 2018). We hypothesized that the observed failure to repair damaged $\mathrm{MCVs}$ would correlate with an increased susceptibility of trafE $\mathrm{KO}$ cells to severe sterile endolysosomal damage caused by LLOMe. To test this, the fluorescent DNA stain propidium iodide (PI), which penetrates dead or dying cells and intercalates and stains their DNA, but is excluded from live cells, was used as a reporter for lytic cell death. In vivo time-lapse confocal microscopy was carried out to compare viability of WT, atgl $\mathrm{KO}$ and trafE $\mathrm{KO}$ cells after $55 \mathrm{~min}$ of high $5 \mathrm{mM}$ LLOMe concentration treatment. Paralleling the results on infected cells (Fig 2E), cells lacking TrafE showed the highest susceptibility to LLOMeinduced sterile perforations (Fig 8D, S3 Movie). These results suggest that the severity of the phenotypes caused by the absence of TrafE are a cumulative consequence of nonfunctional autophagy- and ESCRT-dependent membrane repair.

\section{Loss of TrafE affects the transcription of autophagy and ESCRT gene sets}

To investigate possible explanation of the rapid infection- or LLOMe-induced death of trafE null cells, as well as to identify genes and pathways underlying TrafE-mediated $M$. marinum restriction and endolysosomal homeostasis, we performed a genome-wide transcriptional analysis. RNA of trafE KO mutant cells was collected, sequenced and compared to RNA of WT cells, revealing 283 genes with significantly differential expression (DE) (Fig. 9A, Table S4). Importantly, the steady-state levels of all of the ATGs, including atg1, atg9, atg8a and the autophagy cargo adaptors $p 62$ and $c n r D$, were clearly upregulated (Fig. 9B). They also listed under the KEGG term "autophagy-other", which is significantly enriched by gene set enrichment analysis (GSEA) (Fig. 9C, Table S4 and S5). Earlier studies have demonstrated that high, uncontrolled autophagy activity could be harmful for cells and may lead to accelerated cell death or promote replication and spread of intracellular pathogens or cancer cells (Levine and Yuan, 2005; Ravikumar et al., 2010). In addition, despite that the read counts of the ESCRT genes do not show a clear pattern (Fig. 9B, Table S4), our data showed an overexpression of ALG-2 interacting protein X $(A L I X)$ and downregulation of its putative interacting partner $A \lg 2 A$ (homolog of the human Apoptosis-linked-gene-2). Importantly, it has been demonstrated in human cells, that ALIX overexpression is sufficient to induce cell death, thus establishing a link between the endolysosomal system and the cell death machinery (Mahul-Mellier et al., 2006; Kaul and Chakrabarti, 2017), and that increased association of ALG-2 with ALIX permits increased association of ALIX with membranes (Sun et al., 2015). Enrichment pathway analysis previously performed in our lab following RNA-seq transcriptomic comparison of noninfected or M. marinum-infected D. discoideum cells showed that most of the Biological Processes (BP) of genes upregulated during infection comprise such involved in damage response and cellular defence, particularly in the specific pathways involved in membrane repair (ESCRT) and bacteria elimination (autophagy) (Hanna et al., 2019). Taken together, our data indicates that lack of TrafE alone is sufficient to affect the expression of 
genes belonging to the autophagy and the ESCRT machineries, possibly leading to nonfunctional or severely compromised ESCRT-III and autophagic MCV/endolysosome membrane repair, resulting in drastic reduction of $M$. marinum intracellular growth restriction and precocious cell death.

\section{TrafE is required for optimal ESCRT and autophagy response to membrane damage}

In mammalian and $D$. discoideum cells, LLOMe-induced sterile membrane damage prompts accumulation of $\mathrm{LC} 3 / \mathrm{Atg} 8$ puncta and vigorous recruitment of ALIX and CHMP4B/Vps32 to damaged endolysosomal compartments (López-Jiménez et al., 2019; Mercier et al., 2020). In view of our findings, we asked how the absence of TrafE could impact both the autophagy and the ESCRT machinery responses to sterile endolysosomal damage. To address this question, we generated $D$. discoideum WT or trafE $\mathrm{KO}$ cell lines ectopically expressing GFP-fusions of Atg8a, p62, ALIX or Vps32. We monitored by timelapse $\mathrm{HC}$ confocal microscopy the behaviour of the aforementioned reporter constructs during LLOMe-induced sterile damages. Remarkably, the absence of TrafE impacted the behaviour of all four proteins tested. As expected, the autophagy machinery was recruited to damaged endolysosomes, revealed by as increase in the number of GFP-Atg8a puncta in WT cells but not in trafE KO cells where the number of GFPAtg8a structures remained similar throughout the 40 min period of LLOMe treatment (Fig 10A). In contrast, compared to WT cells, sterile damage in trafE $\mathrm{KO}$ cells resulted in higher levels of GFP-p62 accumulation (Fig 10B), possibly due to inhibition of its degradation (Wu et al., 2020). In WT D. discoideum cells LLOMe treatment caused rapid increase of GFP-Vps32 structures by nearly 10 -fold and unusually high, possibly deleterious increase of GFP-Vps32 structures in trafE KO mutant cells of up to 80-fold (Fig. 10C). Whilst Vps32 was recruited to endolysosomes in a possibly uncontrolled manner, compared to WT, ALIXGFP reacted weakly to LLOMe-induced endolysosomal damage (Fig. 10D). These data provide supportive evidence that TrafE is likely upstream of autophagic and ESCRT-III ELDR. Absence of TrafE leads to non-functional ELDR and drastic reduction of intracellular pathogen restriction.

\section{DISCUSSION}

In recent years, studies in mammals have revealed some of the components of the ELDR signalling pathway that detects, repairs and / or removes intracellular compartments disrupted by invading pathogens or by mechanical damage. Cells have developed molecular sensors that determine the size and the extent of membrane damage, repaired by default by the ESCRT machinery, however extensive and prolonged damage triggers the autophagy machinery. Despite intense research, we still need to extend our insight in the molecular factors involved in membrane damage detection and how it is translated into a vigorous and specific ubiquitynation response. $D$. discoideum is a powerful model system to address the aforementioned questions thanks to its experimental versatility and the high evolutionary conservation of the ESCRT and the autophagy pathways with their mammalian counterparts.

It was previously shown that in $D$. discoideum, $M$. marinum infection or LLOMe-induced sterile damage leads to an increase of $\mathrm{Ub}$ and Atg8-positive structures and to recruitment of the ESCRT subunits Tsg101, Vps32 and Vps4, reportedly ahead of the recruitment of autophagy markers p62 and Atg8 at the MCV and endolysosomes (Cardenal-Muñoz et al., 2017; López-Jiménez et al., 2019). In this study, we demonstrate that early after infection the $D$. discoideum E3 ubiquitin ligase TrafE is recruited to MCVs in a membrane damagedependent manner (Fig 1A, 1B, 1E, 3A and 3B). This recruitment was significantly attenuated upon infection with $M$. marinum $\triangle \mathrm{RD} 1$ (Fig $3 \mathrm{~A}$ and $3 \mathrm{~B}$ ) that produces PDIMs but lacks a functional ESX-1 secretion system. We also demonstrate that the intracellular growth of $M$. marinum-lux is significantly increased in atgl $\mathrm{KO}$ and trafE $\mathrm{KO}$ cells compared to WT cells (Fig 2A), suggesting that autophagy is the main $M$. marinum cellautonomous restriction pathway. In addition, cells lacking the E3 ligase TrafE show exceptionally high and early susceptibility to infection with WT M. marinum which is not the case in the autophagy-deficient atgl KO cells (Fig 2E). Upon starvation, atgl $\mathrm{KO}$ cells that are fully defective in autophagy, display severe developmental phenotypes and do not proceed beyond aggregation (Otto et al., 2004). In contrast, trafE KO cells successfully complete 
their developmental cycle and form spores (Fig. S5), emphasizing on the functional specificity of TrafE to ELDR. Altogether, our data indicate that trafE KO phenocopies the autophagydeficient atg1 mutants in terms of unrestricted $M$. marinum intracellular growth, however the higher cytotoxicity exerted by bacteria in trafE $\mathrm{KO}$ cells compared to WT and atgl KO cells (Fig 7A and 7B) leads to the speculation that TrafE might be upstream of both ESCRT- and autophagy-mediated membrane repair. Following that hypothesis, the particularly rapid death of infected trafE $\mathrm{KO}$ cells likely results from inefficient MCV repair, and leads to early bacteria escape and release by host cell lysis (Fig 2B and 2D).

Ubiquitin is required for various aspects of autophagy, such as removal of protein aggregates (aggrephagy), recycling of mitochondria (mitophagy) and bacteria (xenophagy), or endolysosomal homeostasis (lysophagy). Xenophagy depends on decoration of intracellular pathogens with K63-linked Ub chains that bring bacteria to the phagophore membrane via autophagy cargo receptors such as p62 and NDP52. We demonstrated that although the levels of polyubiquitin-positive MCVs remain unaffected in M. marinuminfected trafE KO cells, the levels of K63linked polyUb-positive MCVs are significantly decreased but not abolished compared to wt $D$. discoideum cells (Fig 4A and 4B), possibly due to the presence of other E3-ligases, such as for instance TrafC, which is also recruited to MCVs during infection and might have partially redundant functions (S1B Fig). It has been shown that human TRAF6 forms heterodimers with TRAF2, TRAF3 and TRAF5 through their RING domains and that the resulting heterodimers are more stable than the TRAF6 homodimers (Das et al., 2021). Therefore, the severity of the trafE KO phenotypes during infection may be due to the instability or non-functionality of a potential heterodimerization redundant partner(s), such as other DdTRAFs. Furthermore, we found that the recruitment of TrafE to MCVs is abolished in the absence of its RING domain and not when the TRAF domain is deleted (Fig 5A), likely because RING domains-mediated dimerization confers the specificity to the substrate via TrafE interaction with the E2conjugating enzyme (Middleton et al., 2017; Kiss et al., 2021). Nevertheless, both domains are equally important for the restriction of $M$. marinum intracellular growth (Fig 5B).

Similar to xenophagy, lysophagy also depends on $\mathrm{Ub}$ and it has been shown that ubiquitination of Lysosome-Associated Membrane Protein 2 (LAMP2) in damaged lysosomes leads to their autophagic degradation (Papadopoulos et al., 2017). In addition, recent studies in mammalian cells have demonstrated that disrupted lysosomes leak protons and reactive oxygen species that lead to pyroptotic cell death (Papadopoulos et al., 2020). Repair of damaged lysosomes is vital for the cells and depends both on the ESCRT machinery and lysophagy, which repair and / or remove extensively damaged compartments (López-Jiménez et al., 2019; Daussy and Wodrich, 2020). Studies in HeLa Kyoto cells have demonstrated that membrane tension plays a role in endosomal membrane remodelling and that both hyperosmotic shock and LLOMe induced perforations, result in a decrease of membrane tension sufficient to trigger the recruitment of the ESCRT-III subunit CHMP4B (Mercier et al., 2020). Remarkably, we found that TrafE is recruited to endolysosomes minutes after LLOMe treatment or hyperosmotic shock (Fig $6 \mathrm{~A}$ and $8 \mathrm{~A}$ ), suggesting its involvement in endolysosomal membrane remodelling and repair signalling pathways. We also demonstrate that, unlike WT D. discoideum cells, TrafE mutants fail to restore their endolysosomal $\mathrm{pH}$ after LLOMe-induced sterile damage (Fig 7A and 7B). Furthermore, as observed during $M$. marinum infection, trafE KO mutant cells are extremely susceptible to LLOMe compared to atgl KO or WT cells (Fig 8D), in agreement with our hypothesis that TrafE is upstream of ESCRT and autophagic ELDR pathways. Comparison of total RNA extracted from WT and trafE KO cells under basal conditions indicated overexpression of nearly all genes belonging to the autophagy pathway (Fig. 9B, Table S4), which in human cells is essential for reactive oxygen species, DNA damage, organelle damage or intracellular pathogen related stress response (Kroemer et al., 2010). The transcriptomic analysis of trafE KO cells also revealed upregulation (Table S4) of a number of $D$. discoideum homologs of the human heat shock $70 \mathrm{kDa}$ protein (HSP70) involved in various cellular stress responses and protection against stress-induced cell death. Furthermore, despite 
an upregulation of the ESCRT-III adapter $A L I X$, our RNA-seq data indicated downregulation of pefA which is $D$. discoideum homolog of the human $A \lg 2 A$ (Fig. 9A), required for recruitment of ALIX to plasma membrane perforations (Scheffer et al., 2014). To investigate further our working model that TrafE is upstream of ESCRT and autophagic ELDR pathways, we monitored the behaviour of the ESCRT-III adapter Alix, the ESCRT-III subunit $\mathrm{Vps} 32$ or the autophagy proteins Atg8a and p62 in trafE KO cells compared to WT cells after LLOMe treatment and found that the recruitment of GFP-Atg8a, GFP-p62, GFPVps32 and Alix-GFP is deregulated (Fig 10A 10D). Taken together, our results suggest that TrafE plays a master role upstream of both autophagy- and ESCRT-III-coordinated membrane damage repair. As a consequence, absence of TrafE leads to non-functional ELDR, rapid infection- or sterile damagerelated cell death and unrestricted $M$. marinum proliferation.

\section{MATERIALS AND METHODS}

Dictyostelium discoideum strains, culture and plasmids. $D$. discoideum $\mathrm{Ax} 2(\mathrm{Ka}) \mathrm{WT}$ and mutant strains, listed in Table S1A, were grown in axenic conditions at $22^{\circ} \mathrm{C}$ in HL5c medium (Formedium) supplemented with $100 \mathrm{U} / \mathrm{mL}$ penicillin and $100 \mu \mathrm{g} / \mathrm{mL}$ streptomycin (Invitrogen) to avoid contamination. Cell lines expressing fluorescent reporters and KO cell lines were cultured in the presence of the appropriate antibiotics, hygromycin (50 $\mu \mathrm{g} / \mathrm{mL})$, blasticidin $(5 \mu \mathrm{g} / \mathrm{mL})$ or Geneticin G418 (5 $\mu \mathrm{g} / \mathrm{mL})$. D. discoideum WT, trafE knock-out (trafE KO) or atgl KO cell lines were stably transformed with GFP-TrafA, GFP-TrafB, GFP-TrafC, GFP-TrafD, GFPTrafE, GFP-Atg8a, GFP-p62, GFP-Vps32, mCherry-Plin constructs (Table S1A). The trafe $\mathrm{KO}$ was generated in $\mathrm{Ax} 2(\mathrm{Ka})$ background by homologous recombination following a one-step cloning in pKOSG-IBAdicty1 (Table S1A) as previously described (Wiegand et al., 2011). The trafE GFP knockin (TrafE-GFP KI) was generated in Ax2(Ka) background by homologous recombination following two-step cloning in pPI183 (Table S1A) (Paschke et al., 2018).
Mycobacterium marinum strains, culture and plasmids. $M$. marinum $\mathrm{WT}$ and $\triangle \mathrm{RD} 1$ strains (Table S1B) expressing GFP, mCherry or a bacterial luciferase operon (Carroll et al., 2010; Andreu et al., 2010; Cardenal-Muñoz et al., 2017) were cultured in shaking at $150 \mathrm{rpm}$ at $32^{\circ} \mathrm{C}$ in Middlebrook $7 \mathrm{H} 9$ (Difco) supplemented with $10 \%$ oleic acid-albumindextrose-catalase (OADC), $0.2 \%$ glycerol, and $0.05 \%$ Tween 80 . Bacterial clumping was minimized by adding 5-mm glass beads during cultivation. Mutants and plasmid carriers were grown in medium supplemented with hygromycin $(100 \mu \mathrm{g} / \mathrm{ml})$ or kanamycin $(25$ $\mu \mathrm{g} / \mathrm{ml})$, as appropriate.

Infection assay. Infections were performed as previously described (Hagedorn and Soldati, 2007; Arafah et al., 2013; Barisch et al., 2015a). M. marinum bacteria were spinoculated onto adherent $D$. discoideum cells and extracellular bacteria were rinsed off. The infected cells were resuspended in $\mathrm{HL} 5 \mathrm{c}$ containing $5 \mathrm{U} \mathrm{mL}^{-1}$ of penicillin and $5 \mu \mathrm{g} \mathrm{mL} \mathrm{m}^{-1}$ of streptomycin (Invitrogen) to prevent extracellular bacterial growth. Infections were performed at a multiplicity of infection (MOI) of 10 for $M$. marinum WT in $D$. discoideum WT. MOI used for M. marinum $\triangle \mathrm{RD} 1$ mutant cells was double the MOI of M. marinum WT. Infected cells for time points analysis were incubated at $25^{\circ} \mathrm{C}$.

Intracellular bacteria growth assay. Measurements of luciferase-expressing $M$. marinum intracellular growth were carried out as previously described (Arafah et al., 2013). In this assay, three different dilutions of infected cells $\left(0.5-2 \times 10^{\wedge} 5\right.$ cells/well $)$ were plated on non-treated, white F96 MicroWell plates (Nunc) with a gas permeable moisture barrier seal (Bioconcept). Luminescence was measured at 1-hour intervals for 30 to 72 hours at a constant temperature of $25^{\circ} \mathrm{C}$ using a Synergy Mx microplate reader (Biotek).

Live fluorescence microscopy. Live microscopy was performed using inverted $3 \mathrm{i}$ Marianas or Nikon Ti CSU-W1 spinning disk microscopes with a $63 \mathrm{x}$ glycerol / air or $100 \mathrm{x}$ oil objectives. 7-10 slices $0.5 \mu \mathrm{m}$ apart were taken for z-stacks. For time-lapse experiments, images were acquired from non-infected or infected cells at intervals of $1 \mathrm{~min}, 5 \mathrm{~min}$ or at the indicated time points (hpi). Image analysis was performed using Fiji (ImageJ). $D$. discoideum cells were plated at $10^{\wedge} 6 / \mathrm{ml}$ in 35 
mm Glass Bottom Dishes (MatTek) or in 4wells $\mu$-slides (Ibidi). Sterile damage in $D$. discoideum was induced using $4.5-5 \mathrm{mM}$ of LLOMe (Bachem) from a 10-fold concentrated solution that was added 5 min after the start of imaging. To induce a hyperosmotic shock, $2 \mathrm{M}$ sorbitol was added to a final concentration of $200 \mathrm{mM}$, as described previously ( $\mathrm{Na}$ et al., 2007). The lumen of endolysosomal compartments was visualized using 10-15 $\mu \mathrm{g} / \mathrm{mL}$ of $10 \mathrm{kDa}$ Fluor 647 Dextran (Invitrogen) added $3 \mathrm{~h}$ prior to microscopy and washed 10-20 min before visualization of the sample. To detect neutralization of endolysosomes, $1 \mu \mathrm{M}$ Lysosensor Green DND189 (Invitrogen) was added $3 \mathrm{~h}$ prior to microscopy and washed away 10-20 min before visualization of the sample. To distinguish live from dead cells $1 \mu \mathrm{g} / \mathrm{mL}$ of propidium iodide (PI) was added directly to the cell culture immediately prior to time-lapse imaging. For high-content live microscopy, M. marinuminfected WT and trafE KO D. discoideum cells (as described above) were deposited in 96-well black, glass-bottom plates (Perkin Elmer, MA, USA) and left to adhere for $30 \mathrm{~min}$ at room temperature. Images were recorded every $1 \mathrm{~min}$ for 30-60 min using a 10x objective with the ImageXpress Micro XL high-content microscope (Molecular Devices). The number of infected cells and extracellular bacteria or GFP-p62-, GFP-atg8a- and GFP-vps32positive structures were counted using the MetaXpress software (Molecular Devices, CA, USA), and analysed using GraphPad Prism ${ }^{\circledR}$ software.

Antibodies and immunofluorescence. $D$. discoideum cells on coverslips were rapidly fixed by immersion in $-85^{\circ} \mathrm{C}$ ultracold methanol as described previously (Hagedorn et al., 2006). The GFP fluorescent signal was enhanced using anti-GFP rabbit polyclonal antibody (MBL), anti-Ub (FK2) mouse monoclonal antibody (Enzo Life Sciences), anti-K63-linkage-specific mouse monoclonal antibody (Enzo Life Sciences). Nuclei were stained with $1 \mu \mathrm{M}$ DAPI (Invitrogen). Cells were embedded using ProlongGold antifade (Invitrogen). As secondary antibodies we used goat anti-rabbit and anti-mouse coupled to Alexa 488 or Alexa 594 (Invitrogen). Images were acquired with a LSM700 or LSM800 microscope (Zeiss) using an oil 63x objective. Image analysis was performed using ImageJ.
qRT-PCR sample collection and analysis. To monitor trafE mRNA levels, mock- or $M$. marinum-infected WT $D$. discoideum cells were harvested, RNA was extracted, and cDNA was synthesized using the Bio-Rad iScript kit. The mean calculated threshold cycles (CT) were averaged and normalized to the $\mathrm{CT}$ of a reference gene with constant expression (GAPDH). The normalized CT was used to calculate the fold change using the $\Delta \Delta \mathrm{CT}$ method. Relative levels of target mRNA, normalized with respect to an endogenous control (GAPDH), were expressed as $2-\Delta \Delta \mathrm{CT}$ (fold), where $\Delta \mathrm{CT}=\mathrm{CT}$ target gene - CT reference gene $(\mathrm{GAPDH})$, and $\Delta \Delta \mathrm{CT}=\Delta \mathrm{CT}$ studied sample - $\triangle \mathrm{CT}$ calibrator conditions.

RNA extraction. RNA was extracted from cells using the directzol RNA extraction kit (Zymo research) following the manufacturer's instructions for total RNA isolation. To remove contaminating genomic DNA, samples were treated with $0.25 \mathrm{U}$ of DNase I (Zymo) per $1 \mu \mathrm{g}$ of RNA for $15 \mathrm{~min}$ at $25{ }^{\circ} \mathrm{C}$. RNA was quantified using Qubit 4.0 (Invitrogen) and its quality was checked on the Agilent 2100 Bioanalyzer (Agilent Technologies).

Sequencing. Total RNAs were subjected to cDNA synthesis and NGS library construction using the Ovation Universal System (NuGEN Technologies, San Carlos, California, USA). $100 \mathrm{ng}$ of total DNAse I-treated RNA was used for first- and then second-strand cDNA synthesis following the manufacturer's protocol. In order to obtain comparable library size, a double bead cut strategy was applied using the $10 \mathrm{X}$ genomics protocol. cDNA was recovered using magnetic beads with two ethanol washing steps, followed by enzymatic end repair of the fragments. Next, barcoded adapters were ligated to each sample, followed by an enzymatic strand selection step and magnetic bead recovery, as above. rRNAs were targeted for depletion by the addition of custom designed oligonucleotides specific for $D$. discoideum (5S,18S, 28S). To amplify the libraries, 18 cycles of PCR were performed based on QC experiments carried out using RTPCR. The quality of the libraries was monitored by TapeStation (Agilent, High Sensitivity D1000 ScreenTape, \# 5067-5584). Eightplexed samples were pooled in approximately equimolar amounts and run in $50 \mathrm{bp}$ single read 
flow cells (Illumina, \# 15022187) and run on a Hiseq 4000 (Illumina).

\begin{abstract}
Alignment. RNA-seq libraries from infected and mock-treated cells taken at the indicated time points were analyzed in pairwise comparisons. $50 \mathrm{nt}$ single-end reads were mapped to the $D$. discoideum genome (downloaded from dictybase) (Fey et al., 2008) using tophat (version 2.0.13) and bowtie2 (version 2.2.4) softwares. Multi hits were not allowed, by using option --max-multi hits 1 . The other parameters were set to default. The read counts per gene were generated using HTSeq software (version 0.6.1) and the GFF annotation downloaded from dictybase (February 2019). Options for htseq-count were -t exon --stranded $=$ yes $-\mathrm{m}$ union.
\end{abstract}

Analysis of differentially expressed genes. Gene counts were imported to R (version 4.0.3) filtered for remaining ribosomal gene counts (DDB_G0295651, DDB_G0295641, DDB_G0295653, DDB_G0295655, DDB_G0295647, DDB_G0295643, DDB_G0295645, DDB_G0295649, DDB_G0294034) and subsequently analyzed using DESeq2 (version 1.30.1) (Love et al., 2014) employing the HTS filter (Rau et al., 2013). A binary comparison trafE KO versus WT was performed, fold change was calculated using the ashr method (Stephens, 2016) in DESeq2, the $p$-value was corrected using the Benjamini-Hochberg (Benjamini and Hochberg, 1995).

Correction. The resulting false discovery rate (FDR) was used for downstream analysis. Hard thresholds for differentially expressed genes (DE genes) were set at the absolute $\log 2$ fold change $\geq 0.585$ (equals to a fold change of 1.5 ) and FDR $\leq 0.05$.

Pathway analysis of DE genes. Pathway analysis was performed using the $\mathrm{R}$ package clusterProfiler (Yu, 2012) and GO (downloaded from QuickGo, 2021-02-16) and KEGG (22.6.2021) annotations. For GSEA genes were ranked by false discovery rate, a group was considered significant at $\mathrm{p}$-value $\leq$ 0.05 , q-value $\leq 0.1$, group size between 2 and 200, using 10000 permutations.
Heatmap of normalized counts. Gene counts were filtered for remaining ribosomal genes (DDB_G0295651, DDB_G0295653, DDB_G0295641, DDB_G0295647, DDB_G0295655, DDB_G0295645, DDB_G0295643, DDB_G0295649, DDB_G0294034) and subsequently for low expressed genes with the function filterByExpr from the edgeR package (Robinson et al., 2009), then normalized using the vst function from the limma package (Ritchie et al., 2015) and batch corrected for the effect of the experimental date using the removeBatchEffect function from the limma package. Normalized counts were subjected to the heatmap. 2 function from the package gplots (3.1.1), scaling and centering the data for each gene.

Single-cell experiments. Single-cell experiment were performed using the InfectChip device (Delincé et al., 2016) as described (Mottet et al, 2021). Briefly, the device is composed of a microfluidic chip in PDMS with a micropatterned serpentine channel, two holders (one in PMMA and one in aluminium), plastic screws and a micropatterned coverslip. Cells were infected as described above and seeded onto the coverslip. The microfluidic chip was placed onto the PMMA holder and covered with an agarose sheet prepared as explained elsewhere (Mottet et al, 2021). Then, the coverslip was placed onto the aluminium holder and the device was mounted and closed with the screws. A $60 \mathrm{~mL}$ syringe was filled with $35 \mathrm{~mL}$ of HL5c supplemented with $5 \mu \mathrm{g} / \mathrm{mL}$ of streptomycin and $5 \mathrm{U} / \mathrm{mL}$ of penicillin, and connected to the tubing of the microfluidic chip. Imaging was performed with a spinning disk confocal microscope (Intelligent Imaging Innovations Marianas SDC mounted on an inverted microscope (Leica DMIRE2)) with the following parameters: $63 \mathrm{X}$ glycerol objective, transmitted light, excitation with laser lines 488 $\mathrm{nm}$ and $594 \mathrm{~nm}$ and temperature controlled at $25^{\circ} \mathrm{C}$. The loaded InfectChip device was placed on the stage and the syringe placed into a syringe pump (WPI, NE-1000) with a flow rate of $10 \mu \mathrm{L} / \mathrm{min}$. Z stacks of 8 slices of $1.5 \mu \mathrm{m}$ each were acquired every $15 \mathrm{~min}$ using phase contrast and every $1 \mathrm{~h}$ for fluorescence for 20 hours total. Image analysis for the single-cell experiments was performed as follows. Briefly, using the ImageJ software (https://imagej.net/Fiji/Downloads), cells were 
manually tracked, and their time of death was recorded, and the results were presented in the form of a survival curves using the GraphPad Prism ${ }^{\circledR}$ software.

Statistics. Microscopy images were analysed using Fiji ImageJ. Experiments in Figs 1B, 1C, $2 \mathrm{~B}, 3 \mathrm{~B}, 4 \mathrm{C}$ and $4 \mathrm{E}$ were quantified manually. Experiments in Figs 7B and 8C were quantified automatically using Fiji ImageJ Time Series Analizer V3. Experiments in Figs 2C, 2D, 9B, 9C and 9D were quantified using MetaXpress. Plots and statistical tests were performed using GraphPad Prism ${ }^{\circledR}$ software. In all plots, standard error of the mean are shown, unless explicitly mentioned. Two-tailed t-test, ANOVA or Two-way ANOVA were used. Post hoc Fisher's LSD and Tukey's multiple comparison tests were performed when necessary (n.s. : non-significant, $*:$ p-value $\leq$ $0.05, * *$ : p-value $\leq 0.01, * * *:$-value $\leq 0.001$, $* * * *$ : p-value $\leq 0.0001)$.

\section{ACKNOWLEDGMENTS:}

We would like to acknowledge the members of the Bioimaging and ACCESS Platforms of the Faculty of Sciences. We thank Dr. Dimitri Moreau for his help with MetaX-press and Dr. Nabil Hanna for his contribution to the conception and practical realization of the RNA-seq experiment.

\section{AUTHOR CONTRIBUTIONS:}

L.R. conceived and designed experiments and analyses, constructed plasmids, generated GFPfusion, knock-out, knock-in constructs and strains, performed and analyzed the experiments, wrote the paper.

M.M. performed the long-term single-cell experiments in microfluidic jails.

L.R. and M.M. created and L.R. analyzed data for Fig. 2E and 3D.

J.N. analyzed RNA-seq data and designed Fig. 9A, 9B and 9C.

T.S. acquired funding, conceived and supervised the project, reviewed and edited the paper.

\section{REFERENCES}

Andreu, N., Zelmer, A., Fletcher, T., Elkington, P. T., Ward, T. H., Ripoll, J., Parish, T., Bancroft, G. J., Schaible, U., Robertson, B. D. \& Wiles, S. 2010. Optimisation of Bioluminescent
Reporters for Use with Mycobacteria. PLOS ONE, 5, e10777.

Arafah, S., Kicka, S., Trofimov, V., Hagedorn, M., Andreu, N., Wiles, S., Robertson, B. \& Soldati, T. 2013. Setting Up and Monitoring an Infection of Dictyostelium discoideum with Mycobacteria. In: EICHINGER, L. \& RIVERO, F. (eds.) Dictyostelium discoideum Protocols. Totowa, NJ: Humana Press.

Augenstreich, J., Haanappel, E., Ferré, G., Czaplicki, G., Jolibois, F., Destainville, N., Guilhot, C., Milon, A., Astarie-Dequeker, C. \& Chavent, M. 2019. The conical shape of DIM lipids promotes Mycobacterium tuberculosis infection of macrophages. Proceedings of the National Academy of Sciences, 116, 2564925658.

Barisch, C., López-Jiménez, A. T. \& Soldati, T. 2015a. Live Imaging of Mycobacterium marinum Infection in Dictyostelium discoideum. In: PARISH, T. \& ROBERTS, D. M. (eds.) Mycobacteria Protocols. New York, NY: Springer New York.

Barisch, C., Paschke, P., Hagedorn, M., Maniak, M. \& Soldati, T. 2015b. Lipid droplet dynamics at early stages of Mycobacterium marinum infection in Dictyostelium. Cellular Microbiology, 17, 1332-1349.

Benjamini, Y. \& Hochberg, Y. 1995. Controlling the False Discovery Rate: A Practical and Powerful Approach to Multiple Testing. Journal of the Royal Statistical Society: Series B (Methodological), 57, 289-300.

Boyle, K. B. \& Randow, F. 2013. The role of 'eatme' signals and autophagy cargo receptors in innate immunity. Current Opinion in Microbiology, 16, 339-348.

Bradley, J. R. \& Pober, J. S. 2001. Tumor necrosis factor receptor-associated factors (TRAFs). Oncogene, 20, 6482-91.

Cardenal-Muñoz, E., Arafah, S., López-Jiménez, A. T., Kicka, S., Falaise, A., Bach, F., Schaad, O., King, J. S., Hagedorn, M. \& Soldati, T. 2017. Mycobacterium marinum antagonistically induces an autophagic response while repressing the autophagic flux in a TORC1-and ESX-1-dependent manner. PLoS Pathogens, 13, e1006344.

Carlsson, F., Joshi, S. A., Rangell, L. \& Brown, E. J. 2009. Polar Localization of VirulenceRelated Esx-1 Secretion in Mycobacteria. PLOS Pathogens, 5, e1000285.

Carroll, P., Schreuder, L. J., Muwanguzi-Karugaba, J., Wiles, S., Robertson, B. D., Ripoll, J., Ward, T. H., Bancroft, G. J., Schaible, U. E. \& Parish, T. 2010. Sensitive Detection of Gene Expression in Mycobacteria under Replicating and Non-Replicating Conditions Using Optimized Far-Red Reporters. PLOS ONE, 5, e9823. 
Chauhan, S., Kumar, S., Jain, A., Ponpuak, M., Mudd, M. H., Kimura, T., Choi, S. W., Peters, R., Mandell, M., Bruun, J.-A., Johansen, T. \& Deretic, V. 2016. TRIMs and Galectins Globally Cooperate and TRIM16 and Galectin3 Co-direct Autophagy in Endomembrane Damage Homeostasis. Developmental cell, 39, 13-27.

Coers, J. \& Haldar, A. K. 2015. Ubiquitination of pathogen-containing vacuoles promotes host defense to Chlamydia trachomatis and Toxoplasma gondii. Communicative \& Integrative Biology, 8, e1115163.

Das, A., Middleton, A. J., Padala, P., Ledgerwood, E. C., Mace, P. D. \& Day, C. L. 2021. The Structure and Ubiquitin Binding Properties of TRAF RING Heterodimers. Journal of Molecular Biology, 433, 166844.

Daussy, C. F. \& Wodrich, H. 2020. "Repair Me if You Can": Membrane Damage, Response, and Control from the Viral Perspective. Cells, 9, 2042.

Delincé, M. J., Bureau, J.-B., López-Jiménez, A. T., Cosson, P., Soldati, T. \& Mckinney, J. D. 2016. A microfluidic cell-trapping device for single-cell tracking of host-microbe interactions. Lab on a Chip, 16, 3276-3285.

Deretic, V., Saitoh, T. \& Akira, S. 2013. Autophagy in infection, inflammation and immunity. Nat Rev Immunol, 13, 722-37.

Dunn, J. D., Bosmani, C., Barisch, C., Raykov, L., Lefrançois, L. H., Cardenal-Muñoz, E., LópezJiménez, A. T. \& Soldati, T. 2018. Eat Prey, Live: Dictyostelium discoideum As a Model for Cell-Autonomous Defenses. Frontiers in immunology, 8, 1906-1906.

Eriksson, I., Wäster, P. \& Öllinger, K. 2020. Restoration of lysosomal function after damage is accompanied by recycling of lysosomal membrane proteins. Cell Death \& Disease, 11, 370.

Fey, P., Gaudet, P., Curk, T., Zupan, B., Just, E. M., Basu, S., Merchant, S. N., Bushmanova, Y. A., Shaulsky, G., Kibbe, W. A. \& Chisholm, R. L. 2008. dictyBase-a Dictyostelium bioinformatics resource update. Nucleic Acids Research, 37, D515-D519.

Franco, L. H., Nair, V. R., Scharn, C. R., Xavier, R. J., Torrealba, J. R., Shiloh, M. U. \& Levine, B. 2017. The Ubiquitin Ligase Smurfl Functions in Selective Autophagy of Mycobacterium tuberculosis and Anti-tuberculous Host Defense. Cell Host \& Microbe, 21, 59-72.

Gao, L. Y., Guo, S., Mclaughlin, B., Morisaki, H., Engel, J. N. \& Brown, E. J. 2004. A mycobacterial virulence gene cluster extending $\mathrm{RD} 1$ is required for cytolysis, bacterial spreading and ESAT-6 secretion. $\mathrm{Mol}$ Microbiol, 53, 1677-93.
Gómez-Díaz, C. \& Ikeda, F. 2019. Roles of ubiquitin in autophagy and cell death. Seminars in Cell \& Developmental Biology, 93, 125-135.

Guého, A., Bosmani, C. \& Soldati, T. 2019. Proteomic characterization of the Mycobacterium marinum-containing vacuole in Dictyostelium discoideum. bioRxiv, 592717.

Hagedorn, M., Neuhaus, E. M. \& Soldati, T. 2006. Optimized Fixation and Immunofluorescence Staining Methods for Dictyostelium Cells. In: EICHINGER, L. \& RIVERO, F. (eds.) Dictyostelium discoideum Protocols. Totowa, NJ: Humana Press.

Hagedorn, M., Rohde, K. H., Russell, D. G. \& Soldati, T. 2009. Infection by tubercular mycobacteria is spread by nonlytic ejection from their amoeba hosts. Science (New York, N.Y.), 323, 1729-1733.

Hagedorn, M. \& Soldati, T. 2007. Flotillin and RacH modulate the intracellular immunity of Dictyostelium to Mycobacterium marinum infection. Cellular Microbiology, 9, 2716-2733.

Hanna, N., Burdet, F., Melotti, A., Bosmani, C., Kicka, S., Hilbi, H., Cosson, P., Pagni, M. \& Soldati, T. 2019. Time-resolved RNA-seq profiling of the infection of Dictyostelium discoideum by Mycobacterium marinum reveals an integrated host response to damage and stress. bioRxiv, 590810.

Huett, A., Heath, R. J., Begun, J., Sassi, S. O., Baxt, L. A., Vyas, J. M., Goldberg, M. B. \& Xavier, R. J. 2012. The LRR and RING domain protein LRSAM1 is an E3 ligase crucial for ubiquitin-dependent autophagy of intracellular Salmonella Typhimurium. Cell host \& microbe, 12, 778-790.

Huotari, J. \& Helenius, A. 2011. Endosome maturation. The EMBO Journal, 30, 3481-3500.

Jackson, P. K., Eldridge, A. G., Freed, E., Furstenthal, L., Hsu, J. Y., Kaiser, B. K. \& Reimann, J. D. 2000. The lore of the RINGs: substrate recognition and catalysis by ubiquitin ligases. Trends Cell Biol, 10, 429-39.

Joazeiro, C. a. P. \& Weissman, A. M. 2000. RING Finger Proteins: Mediators of Ubiquitin Ligase Activity. Cell, 102, 549-552.

Kaul, Z. \& Chakrabarti, O. 2017. Tumor susceptibility gene 101 regulates predisposition to apoptosis via ESCRT machinery accessory proteins. Molecular biology of the cell, 28, 2106-2122.

Kiss, L., Clift, D., Renner, N., Neuhaus, D. \& James, L. C. 2021. RING domains act as both substrate and enzyme in a catalytic arrangement to drive self-anchored ubiquitination. Nature Communications, 12, 1220.

Koerver, L., Papadopoulos, C., Liu, B., Kravic, B., Rota, G., Brecht, L., Veenendaal, T., Polajnar, M., Bluemke, A., Ehrmann, M., Klumperman, J., Jäättelä, M., Behrends, C. \& Meyer, H. 2019. 
The ubiquitin-conjugating enzyme UBE2QL1 coordinates lysophagy in response to endolysosomal damage. EMBO reports, 20, e48014.

Kosugi, S., Hasebe, M., Tomita, M. \& Yanagawa, H. 2009. Systematic identification of cell cycledependent yeast nucleocytoplasmic shuttling proteins by prediction of composite motifs. Proceedings of the National Academy of Sciences, 106, 10171-10176.

Kroemer, G., Mariño, G. \& Levine, B. 2010. Autophagy and the integrated stress response. Molecular cell, 40, 280-293.

Lerner, T. R., Queval, C. J., Fearns, A., Repnik, U., Griffiths, G. \& Gutierrez, M. G. 2018.

Phthiocerol dimycocerosates promote access to the cytosol and intracellular burden of Mycobacterium tuberculosis in lymphatic endothelial cells. BMC Biol, 16, 1.

Levine, B. \& Yuan, J. 2005. Autophagy in cell death: an innocent convict? J Clin Invest, 115, 2679-88.

Lienard, J., Nobs, E., Lovins, V., Movert, E., Valfridsson, C. \& Carlsson, F. 2020. The Mycobacterium marinum ESX-1 system mediates phagosomal permeabilization and type I interferon production via separable mechanisms. Proceedings of the National Academy of Sciences, 117, 1160.

López-Jiménez, A. T., Cardenal-Muñoz, E., Leuba, F., Gerstenmaier, L., Barisch, C., Hagedorn, M., King, J. S. \& Soldati, T. 2019. The ESCRT and autophagy machineries cooperate to repair ESX-1-dependent damage at the Mycobacterium-containing vacuole but have opposite impact on containing the infection. PLOS Pathogens, 14, e1007501.

Love, M. I., Huber, W. \& Anders, S. 2014. Moderated estimation of fold change and dispersion for RNA-seq data with DESeq2. Genome Biology, 15, 550.

Mahul-Mellier, A.-L., Hemming, F. J., Blot, B., Fraboulet, S. \& Sadoul, R. 2006. Alix, making a link between apoptosis-linked gene-2, the endosomal sorting complexes required for transport, and neuronal death in vivo. The Journal of neuroscience : the official journal of the Society for Neuroscience, 26, 542-549.

Manzanillo, P. S., Ayres, J. S., Watson, R. O., Collins, A. C., Souza, G., Rae, C. S., Schneider, D. S., Nakamura, K., Shiloh, M. U. \& Cox, J. S. 2013. The ubiquitin ligase parkin mediates resistance to intracellular pathogens. Nature, 501, 512-516.

Mercier, V., Larios, J., Molinard, G., Goujon, A., Matile, S., Gruenberg, J. \& Roux, A. 2020. Endosomal membrane tension regulates ESCRT-III-dependent intra-lumenal vesicle formation. Nature Cell Biology, 22, 947-959.
Mesquita, A., Cardenal-Muñoz, E., Dominguez, E., Muñoz-Braceras, S., Nuñez-Corcuera, B., Phillips, B. A., Tábara, L. C., Xiong, Q., Coria, R., Eichinger, L., Golstein, P., King, J. S., Soldati, T., Vincent, O. \& Escalante, R. 2017. Autophagy in Dictyostelium: Mechanisms, regulation and disease in a simple biomedical model. Autophagy, 13, 24-40.

Middleton, A. J., Budhidarmo, R., Das, A., Zhu, J., Foglizzo, M., Mace, P. D. \& Day, C. L. 2017.

The activity of TRAF RING homo- and heterodimers is regulated by zinc finger 1 . Nature Communications, 8, 1788.

Na, J., Tunggal, B. \& Eichinger, L. 2007. STATc is a key regulator of the transcriptional response to hyperosmotic shock. BMC Genomics, 8, 123.

Nguyen Ba, A. N., Pogoutse, A., Provart, N. \& Moses, A. M. 2009. NLStradamus: a simple Hidden Markov Model for nuclear localization signal prediction. BMC Bioinformatics, 10, 202.

Osman, M. M., Pagán, A. J., Shanahan, J. K. \& Ramakrishnan, L. 2020. Mycobacterium marinum phthiocerol dimycocerosates enhance macrophage phagosomal permeabilization and membrane damage. PloS one, 15, e0233252e0233252.

Otto, G. P., Wu, M. Y., Kazgan, N., Anderson, O. R. \& Kessin, R. H. 2004. Dictyostelium macroautophagy mutants vary in the severity of their developmental defects. J Biol Chem, 279, 15621-9.

Papadopoulos, C., Kirchner, P., Bug, M., Grum, D., Koerver, L., Schulze, N., Poehler, R., Dressler, A., Fengler, S., Arhzaouy, K., Lux, V., Ehrmann, M., Weihl, C. C. \& Meyer, H. 2017. $\mathrm{VCP} / \mathrm{p} 97$ cooperates with YOD1, UBXD1 and PLAA to drive clearance of ruptured lysosomes by autophagy. Embo j, 36, 135-150.

Papadopoulos, C., Kravic, B. \& Meyer, H. 2020. Repair or Lysophagy: Dealing with Damaged Lysosomes. Journal of Molecular Biology, 432, 231-239.

Papadopoulos, C. \& Meyer, H. 2017. Detection and Clearance of Damaged Lysosomes by the EndoLysosomal Damage Response and Lysophagy. Curr Biol, 27, R1330-r1341.

Park, H. H. 2018. Structure of TRAF Family: Current Understanding of Receptor Recognition. Frontiers in immunology, 9, 19991999.

Paschke, P., Knecht, D. A., Silale, A., Traynor, D., Williams, T. D., Thomason, P. A., Insall, R. H., Chubb, J. R., Kay, R. R. \& Veltman, D. M. 2018. Rapid and efficient genetic engineering of both wild type and axenic strains of Dictyostelium discoideum. PLoS One, 13, e0196809.

Pei, G., Buijze, H., Liu, H., Moura-Alves, P., Goosmann, C., Brinkmann, V., Kawabe, H., Dorhoi, A. \& Kaufmann, S. H. E. 2017. The E3 
ubiquitin ligase NEDD4 enhances killing of membrane-perturbing intracellular bacteria by promoting autophagy. Autophagy, 13, 20412055.

Pym, A. S., Brodin, P., Brosch, R., Huerre, M. \& Cole, S. T. 2002. Loss of RD1 contributed to the attenuation of the live tuberculosis vaccines Mycobacterium bovis BCG and Mycobacterium microti. Mol Microbiol, 46, 709-17.

Queval, C. J., Brosch, R. \& Simeone, R. 2017. The Macrophage: A Disputed Fortress in the Battle against Mycobacterium tuberculosis. Frontiers in Microbiology, 8.

Quigley, J., Hughitt, V. K., Velikovsky, C. A., Mariuzza, R. A., El-Sayed, N. M. \& Briken, V. 2017. The Cell Wall Lipid PDIM Contributes to Phagosomal Escape and Host Cell Exit of Mycobacterium tuberculosis. mBio, 8 .

Randow, F. \& Youle, R. J. 2014. Self and nonself: how autophagy targets mitochondria and bacteria. Cell Host Microbe, 15, 403-11.

Rau, A., Gallopin, M., Celeux, G. \& Jaffrézic, F. 2013. Data-based filtering for replicated highthroughput transcriptome sequencing experiments. Bioinformatics, 29, 2146-2152.

Ravikumar, B., Sarkar, S., Davies, J. E., Futter, M., Garcia-Arencibia, M., Green-Thompson, Z. W., Jimenez-Sanchez, M., Korolchuk, V. I., Lichtenberg, M., Luo, S., Massey, D. C., Menzies, F. M., Moreau, K., Narayanan, U., Renna, M., Siddiqi, F. H., Underwood, B. R., Winslow, A. R. \& Rubinsztein, D. C. 2010. Regulation of mammalian autophagy in physiology and pathophysiology. Physiol Rev, 90, 1383-435.

Repnik, U., Borg Distefano, M., Speth, M. T., Ng, M. Y. W., Progida, C., Hoflack, B., Gruenberg, J. \& Griffiths, G. 2017. L-leucyl-L-leucine methyl ester does not release cysteine cathepsins to the cytosol but inactivates them in transiently permeabilized lysosomes. Journal of Cell Science, 130, 3124-3140.

Ritchie, M. E., Phipson, B., Wu, D., Hu, Y., Law, C. W., Shi, W. \& Smyth, G. K. 2015. limma powers differential expression analyses for RNA-sequencing and microarray studies. Nucleic Acids Research, 43, e47-e47.

Robinson, M. D., Mccarthy, D. J. \& Smyth, G. K. 2009. edgeR: a Bioconductor package for differential expression analysis of digital gene expression data. Bioinformatics, 26, 139-140.

Sattler, U. G. A., Walenta, S. \& Mueller-Klieser, W. 2007. A bioluminescence technique for quantitative and structure-associated imaging of pyruvate. Laboratory Investigation, 87, 84-92.

Scheffer, L. L., Sreetama, S. C., Sharma, N., Medikayala, S., Brown, K. J., Defour, A. \& Jaiswal, J. K. 2014. Mechanism of $\mathrm{Ca}^{2+}$ triggered ESCRT assembly and regulation of cell membrane repair. Nature communications, 5, 5646-5646.

Schuck, S. 2020. Microautophagy - distinct molecular mechanisms handle cargoes of many sizes. Journal of Cell Science, 133, jcs246322.

Sharma, V., Verma, S., Seranova, E., Sarkar, S. \& Kumar, D. 2018. Selective Autophagy and Xenophagy in Infection and Disease. Frontiers in Cell and Developmental Biology, 6.

Skowyra, M. L., Schlesinger, P. H., Naismith, T. V. \& Hanson, P. I. 2018. Triggered recruitment of ESCRT machinery promotes endolysosomal repair. Science, 360, eaar5078.

Soldati, T. \& Neyrolles, O. 2012. Mycobacteria and the intraphagosomal environment: take it with a pinch of salt(s)! Traffic, 13, 1042-52.

Stajdohar, M., Rosengarten, R. D., Kokosar, J., Jeran, L., Blenkus, D., Shaulsky, G. \& Zupan, B. 2017. dictyExpress: a web-based platform for sequence data management and analytics in Dictyostelium and beyond. $B M C$ Bioinformatics, 18, 291.

Stephens, M. 2016. False discovery rates: a new deal. Biostatistics, 18, 275-294.

Sun, S., Zhou, X., Corvera, J., Gallick, G. E., Lin, S.-H. \& Kuang, J. 2015. ALG-2 activates the MVB sorting function of ALIX through relieving its intramolecular interaction. Cell Discovery, 1, 15018.

Vietri, M., Radulovic, M. \& Stenmark, H. 2020. The many functions of ESCRTs. Nature Reviews Molecular Cell Biology, 21, 25-42.

Wang, F., Gómez-Sintes, R. \& Boya, P. 2018. Lysosomal membrane permeabilization and cell death. Traffic, 19, 918-931.

Wiegand, S., Kruse, J., Gronemann, S. \& Hammann, C. 2011. Efficient generation of gene knockout plasmids for Dictyostelium discoideum using one-step cloning. Genomics, 97, 321-5.

Wu, Y., Jin, Y., Sun, T., Zhu, P., Li, J., Zhang, Q., Wang, X., Jiang, J., Chen, G. \& Zhao, X. 2020. p62/SQSTM1 accumulation due to degradation inhibition and transcriptional activation plays a critical role in silica nanoparticle-induced airway inflammation via NF- $\mathrm{B}$ activation. Journal of Nanobiotechnology, 18, 77.

Xie, P. 2013. TRAF molecules in cell signaling and in human diseases. Journal of Molecular Signaling, 8, 7-7.

Yin, Q., Lin, S.-C., Lo, Y.-C., Damo, S. M. \& Wu, H. 2010. Chapter 49 - Tumor Necrosis Factor Receptor-Associated Factors in Immune Receptor Signal Transduction A2 - Bradshaw, Ralph A. In: DENNIS, E. A. (ed.) Handbook of Cell Signaling (Second Edition). San Diego: Academic Press.

Yoshida, Y., Yasuda, S., Fujita, T., Hamasaki, M., Murakami, A., Kawawaki, J., Iwai, K., Saeki, Y., Yoshimori, T., Matsuda, N. \& Tanaka, K. 
2017. Ubiquitination of exposed glycoproteins by SCF FBXO27 directs damaged lysosomes for autophagy. Proceedings of the National Academy of Sciences, 114, 8574.

\section{LEGENDS OF FIGURES SUPPLEMENTARY FIGURES} AND

Figure 1. Upon infection TrafE is overexpressed and recruited to MCVs. (A) GFP-TrafE-expressing $D$. discoideum cells (green) were mock-infected or infected for 1.5, 3 , 6 or $24 \mathrm{~h}$ with mCherry-expressing or DsRed-expressing $M$. marinum (red). Representative maximum projections of live images with arrowheads pointing at GFP-TrafE recruitment to $\mathrm{MCVs} /$ bacteria. Scale bars correspond to $10 \mu \mathrm{m}$. Images are representative of at least 3 independent experiments. (B) Quantification of the percentage of intracellular $\mathrm{MCV}$ /bacteria positive for GFP-TrafE during the infection time-course. Bars represent SEM from 3 independent experiments. (C) Quantification of GFP-TrafE in the vicinity of $M$. marinum poles from random images in 3 independent experiments. (D) Normalized mRNA levels of trafE in mock-infected or $M$. marinum-infected $D$. discoideum cells at 1.5, 6 or 24 hours. Shown are mean and SEM from 3 independent experiments. Statistical differences were calculated with an unpaired $t$ test (n.s. : non-significant, $*: p \leq 0.05$ ). (E) The intracellular growth of $M$. marinum-lux was monitored every hour inside WT and TrafE overexpressing cells. Error bars represent SEM. Statistical difference from 2 independent experiments were calculated using Bonferroni multiple comparison test after ANOVA $(* * *$ : $p \leq 0.001$ ). (F) Recruitment of the endogenous TrafE-GFP (green) to MCV/bacteria (red) after 1.5 hpi. Scale bars correspond to $10 \mu \mathrm{m}$. Images are representative of at least 3 independent experiments.

Figure 2. Loss of TrafE is detrimental for $D$. discoideum cells after infection with $M$. marinum. (A) The intracellular growth of $M$. marinum-lux was monitored every 1 hour inside WT, atgl KO, trafE KO or GFP-TrafE complemented trafE KO cells. Error bars

represent SEM. Statistical difference from 2-4 independent experiments were calculated using Bonferroni multiple comparison test after ANOVA $^{* * *}: \mathrm{p} \leq 0.001$ ). (B) WT, atgl KO or
Yu, G. 2012. clusterProfiler: an R Package for Comparing Biological Themes Among Gene Clusters. OMICS: A Journal of Integrative Biology, 16, 284-287.

trafE KO cells expressing mCherry-Plin were infected with GFP-expressing M. marinum. The percentage of cells with intracellular bacteria colocalizing with GFP/RFP Plin was assessed manually at 6 and 24 hpi. Error bars indicate the SEM from 3 independent experiments. Statistical differences are indicated with an asterisk and were calculated with an unpaired $t$ test (n.s. : non-significant, $*: \mathrm{p} \leq 0.05, * *$ : $\mathrm{p}$ value $\leq 0.01, * * *: \mathrm{p} \leq 0.001)$. (C-D) WT or trafE $\mathrm{KO}$ cells infected with DsRed-expressing $M$. marinum imaged by high-content microscopy every $1 \mathrm{~h}$. Number of infected cells (C) or number of extracellular bacteria (D) were monitored and the average of 3 replicates $\mathrm{n} \geq 200$ cells per time point was plotted. Error bars represent SEM. Statistical differences were calculated using Bonferroni multiple comparison test after two-way ANOVA (*** : $\mathrm{p} \leq 0.001$ ). (E) WT, atgl KO, trafE KO or GFP-TrafE-complemented trafE KO cells infected with mCherry-expressing M. marinum in microfluidic chip single-cell experiment were monitored every $1 \mathrm{~h}$. The number of live cells was counted at each time point for $20 \mathrm{~h}$ and the counts from 3 independent experiments were plotted as Kaplan-Meier survival curves. Statistical differences were calculated using Bonferroni multiple comparison test after twoway ANOVA (n.s. : non-significant, $* * *: \mathrm{p} \leq$ $0.001)$.

Figure 3. TrafE recruitment to MCVs/bacteria is membrane damagedependent. (A) D. discoideum cells expressing TrafE-GFP (green) were infected for 1.5, 3 or $24 \mathrm{~h}$ with M. marinum WT (red) or M. marinum $\triangle \mathrm{RD1}$ (red). Representative maximum projections of live images with arrowheads pointing at TrafE-GFP recruitment to $\mathrm{MCV}$ s/bacteria. Scale bars correspond to 10 $\mu \mathrm{m}$. Images are representative of 3 independent experiments. (B) Quantification of the percentage of intracellular MCV/bacteria positive for TrafE-GFP during the infection time-course. SEM from 2-4 independent experiments. Statistical differences were calculated with an unpaired t test $(* * * *: \mathrm{p}$ value $\leq 0.0001)$. (C) ) The intracellular growth of $M$. marinum-lux WT or $M$. marinum $\triangle \mathrm{RD} 1$ 
was monitored every hour inside WT or trafE KO cells. Error bars represent SEM. Statistical differences from 2 independent experiments were calculated using Bonferroni multiple comparison test after one-way ANOVA (*** : $\mathrm{p} \leq 0.001$ ). (D) WT, atgl KO, trafE $\mathrm{KO}$ or GFP-TrafE-complemented trafE KO cells infected with $M$. marinum-lux $\triangle \mathrm{RD} 1$ in microfluidic chip single-cell experiment were monitored every $1 \mathrm{~h}$. The number of live cells was counted at each time point for $20 \mathrm{~h}$ and the counts from 3 independent experiments were plotted as Kaplan-Meier survival curves. Statistical difference were calculated using Bonferroni multiple comparison test after ANOVA (n.s. : non-significant).

Figure 4. K-63-linked polyubiquitination decrease in D. discoideum trafE KO cells. (A) Maximum projections showing colocalization (white arrowheads) of M. marinum (blue), K63linked polyubiquitin chains (grey) and GFPTrafE (green). Maximum projections showing (B) polyubiquitin (grey) or (D) K63-linked polyubiquitin (grey) colocalization (white arrowheads) with $M$. marinum. Scale bars correspond to $10 \mu \mathrm{m}$. Quantification of the percentage of (C) FK2-positive or (E) K63positive intracellular $\mathrm{MCV} /$ bacteria at $6 \mathrm{hpi}$ was carried out manually from 10-15 images per experiment $n \geq 200$ cells. Error bars indicate SEM. Statistical differences were calculated with an unpaired t test (n.s. : non-significant, $* *$ $: \mathrm{p} \leq 0.01)$.

Figure 5. TrafE recruitment to $\mathrm{MCV}$ is RING domain-dependent. (A) D. discoideum trafE KO cells expressing GFP-TrafE, GFPTrafE with truncated RING domain or GFPTrafE with truncated N-terminal TRAF domain (green) were infected for 1.5, 3, 6 or $24 \mathrm{~h}$ with mCherry-expressing $M$. marinum (red). Representative maximum projections of live images with arrow heads pointing at GFPTrafE recruitment to MCVs/bacteria. Scale bars correspond to $10 \mu \mathrm{m}$. Images are representative of 3 independent experiments. (B) The intracellular growth of $M$. marinum-lux was monitored every hour inside WT, trafE KO or D. discoideum cells expressing GFP-TrafE with truncated RING domain or GFP-TrafE with truncated N-terminal TRAF domain. Error bars represent SEM. Statistical differences from 2-4 independent experiments were calculated using Bonferroni multiple comparison test after
ANOVA (n.s. : non-significant, $* * *: p \leq$ $0.001)$.

Figure 6. LLOMe-induced damage triggers TrafE recruitment to endolysosomes. $D$. discoideum trafE KO cells expressing (A) GFPTrafE, (B) GFP-TrafE with truncated RING domain or (C) GFP-TrafE with truncated Nterminal TRAF domain (green) were treated with $4.5 \mathrm{mM}$ LLOMe $5 \mathrm{~min}(\mathrm{t}=0)$ after $25 \mathrm{~min}$ live microscopy time-course. Images were taken every $1 \mathrm{~min}$. White rectangles indicate zoom of selected areas. Images are representative of at least 3 independent experiments. Scale bars correspond to $10 \mu \mathrm{m}$. (D) Colocalization of GFP-TrafE (green) with Alexa Fluor $64710 \mathrm{kDa}$ dextran (red) $10 \mathrm{~min}$ after LLOMe treatment. Images are representative of at least 3 independent experiments. Scale bars correspond to $10 \mu \mathrm{m}$.

Figure 7. Absence of TrafE impacts the repair of damaged compartments. (A) Maximum projection confocal images of live D. discoideum WT or trafE KO cells incubated with Lysosensor Green DND-189 (green), imaged every $1 \mathrm{~min}, 5 \mathrm{~min}$ before and $30 \mathrm{~min}$ after addition of $4.5 \mathrm{mM}$ LLOMe. Images are representative of 3 independent experiments. Scale bars correspond to $10 \mu \mathrm{m}$. (B) Quantification of $\mathrm{pH}$ tracer Lysosensor Green DND-189 (green) fluorescence average intensity using ImageJ Time Series Analyzer V3. Error bars represent SEM. Statistical differences were calculated using Bonferroni multiple comparison test after two-way $\operatorname{ANOVA}(* * *: p \leq 0.001)$.

Figure 8. TrafE is relocalized to endolysosomes following hypertonic shock. (A) Maximum projection of confocal images of D. discoideum cells expressing GFP-TrafE (green) imaged every $1 \mathrm{~min}, 5 \mathrm{~min}$ before and $55 \mathrm{~min}$ after addition of $200 \mathrm{mM}$ sorbitol Images are representative of at least 3 three experiments. Scale bars correspond to $10 \mu \mathrm{m}$. (B) Maximum projection of confocal images of live $D$. discoideum WT cells incubated with Lysosensor Green DND-189 (green) and imaged every $1 \mathrm{~min}, 5 \mathrm{~min}$ before and $30 \mathrm{~min}$ after addition of $4.5 \mathrm{mM}$ LLOMe or $200 \mathrm{mM}$ sorbitol. Images are representative of 3 independent experiments. Scale bars correspond to $10 \mu \mathrm{m}$. (C) Quantification of fluorescence average intensity of $\mathrm{pH}$ tracer 
Lysosensor Green DND-189 (green), before and after LLOMe or Lysosensor addition, using ImageJ Time Series Analyzer V3. Error bars represent SEM. Statistical difference were calculated using Bonferroni multiple comparison test after two-way ANOVA (*** : $\mathrm{p} \leq 0.001$ ). (D) WT, atgl KO or trafE KO cells were incubated in propidium iodide (PI) and monitored by live confocal time-lapse microscopy every $1 \mathrm{~min} 5 \mathrm{~min}$ before and 55 min after addition of $5 \mathrm{mM}$ LLOMe. Number of PI-positive cells at min 55 were counted from 4 independent experiments. Error bars represent SEM. Statistical differences were calculated with an unpaired t test (n.s. : nonsignificant, $*: p \leq 0.05, * *: p \leq 0.01)$

Figure 9. RNA-seq results. (A) Volcano plot of Differential Expression (DE) genes comparing trafE KO to WT. After filtering for remaining ribosomal gene reads and low expressed genes, 8744 genes were left for the DE genes analysis, of which 283 were DE (absolute $\log 2 \mathrm{fc} \geq 0.585$, FDR $\leq 0.05$ ). From these, 144 were upregulated (red) and 139 were downregulated (blue). For illustrational purposes, trafE was included, which is DE and downregulated (blue dot on the far left), which is a confirmation of the trafE clean $\mathrm{KO}$ on transcriptional level. (B) Heatmap of normalized read counts. Filtered and normalized read counts were interrogated for a previously defined list of genes of interest. This included a set associated with autophagy (upper segment) and a set associated with the ESCRT machinery (lower segment). Biological replicates are depicted as three columns per condition (WT and trafE KO). A clear pattern of upregulation is visible in autophagy-related genes, whereas no clear overall pattern is visible for ESCRT-related genes. However, key players in the ESCRT machinery such as alxA show a striking pattern and most notably, $\mathrm{Ca}^{2+}$ dependent regulators pefA and pefB are regulated inversely. None of the genes depicted are $\mathrm{DE}$ by the statistical criteria defined previously. (C) Gene set enrichment analysis (GSEA) of $\log 2$ fold change of filtered genes. For GSEA, genes were ranked by $\log 2$ fold change, resulting groups were restricted by minimum and maximum group size of respectively 2 and 200 and considered significant at a $p$-value $\leq 0.05$ and a $q$-value $\leq$ 0.1 . Depicted are the $\log 2$ fold change density distributions for enriched core genes in each respective term. The significant signature included several metabolic terms including Proteasome, Endocytosis and most notably Autophagy as being upregulated.

Figure 10. TrafE directs ESCRT and autophagy ELDR. (A-D) WT or trafE KO cells expressing GFP-fusions of Atg8a, p62, Vps32 or Alix were monitored by time-lapse high-content confocal microscopy, imaged every $1 \mathrm{~min} 5 \mathrm{~min}$ before and $40 \mathrm{~min}$ after treatment with $4.5 \mathrm{mM}$ LLOMe. Number of (A) Atg8a, (B) p62, (C) Vps32 or (D) Alix structures were quantified from the average of 3 independent experiments $n \geq 200$ cells per time point and experiment are plotted. Bars represent SEM. Statistical differences were calculated using Bonferroni multiple comparison test after two-way ANOVA $(* * *: \mathrm{p} \leq 0.001)$.

Supplementary Table 1. D. discoideum material used in this study. The table includes the $D$. discoideum strains used in this study, the overexpression plasmids and the plasmids used for generation of trafE knock-out and GFP knock-in.

Supplementary Table 2. M. marinum material used in this study. The table includes all $M$. marinum strains and M. marinum plasmids used in this study.

Supplementary Table 3. Primers used in this study. The table includes all primers used to amplify trafA, trafB, trafC and trafE CDSs, primers used for knock-out, GFP knock-in generation and primers for screens.

Supplementary Table 4. Differential Expression (DE) genes results for the $D$. discoideum WT and trafE KO cells transcriptome analyzis.

Supplementary Table 5. Gene set enrichment analysis (GSEA) on $\log 2$ fold change of filtered genes and statistics output.

Supplementary Figure 1. trafE is upregulated early after infection. RNAsequencing of non-infected and M. marinuminfected $D$. discoideum cells. RNA levels were compared and the fold change (FC) was plotted.

Supplementary Figure 2. TrafA and TrafB do nor relocalize after infection with $M$. 
marinum. (A) GFP-TrafA-or (B) GFP-TrafB expressing $D$. discoideum cells (green) were mock-infected or infected for $1.5,3,6$ or $24 \mathrm{~h}$ with mCherry-expressing or DsRed-expressing M. marinum (red). Representative maximum projections of live images of at least 3 independent experiments. Scale bars correspond to $10 \mu \mathrm{m}$.

Supplementary Figure 3. Despite their similarity TrafC and TrafD display different behaviour after infection with $M$. marinum. (A) GFP-TrafC-or (B) GFP-TrafD expressing $D$. discoideum cells (green) were mockinfected or infected for $1.5,3,6$ or $24 \mathrm{~h}$ with mCherry-expressing or DsRed-expressing $M$. marinum (red). Representative maximum projections of live images of at least 3 independent experiments. Scale bars correspond to $10 \mu \mathrm{m}$.

Supplementary Figure 4. M. marinum growth is not affected by TrafC, TrafD overexpression and TrafE C-terminal GFP tagging. (A) The intracellular growth of $M$. marinum-lux was monitored every hour inside WT, TrafC or TrafD overexpressing cells. Error bars represent SEM. Statistical differences from 3 independent experiments were calculated using Bonferroni multiple comparison test after ANOVA (n.s. : nonsignificant). (B) The intracellular growth of $M$. marinum-lux was monitored every hour inside WT or TrafE-GFP KI cells. Error bars represent SEM. Statistical differences from 3 independent experiments were calculated using Bonferroni multiple comparison test after ANOVA (n.s. : non-significant).
Supplementary Figure 5. Development of $\boldsymbol{D}$. discoideum. WT, atgl $\mathrm{KO}$ and trafE $\mathrm{KO}$ cells were deposited on Soerensen-agar plates and visualized by microscopy after $24 \mathrm{~h}$ of starvation. WT and trafE KO cells aggregate and form fruiting bodies, whereas atgl $\mathrm{KO}$ cells do not aggregate and do not progress to the formation of fruiting bodies.

Movie S1. TrafE relocalization to endolysosomal compartments upon LLOMe treatment. Live microscopy time-course of $D$. discoideum trafE KO cells expressing GFPTrafE, treated with $4.5 \mathrm{mM}$ LLOMe. Images were taken every $1 \mathrm{~min}, 5 \mathrm{~min}$ before $(\mathrm{t}=0)$ and $55 \mathrm{~min}$ after LLOMe treatment. Images are representative of at least 3 independent experiments. Scale bars correspond to $10 \mu \mathrm{m}$.

Movie S2. TrafE colocalizes with $10 \mathrm{kDa}$ dextran upon LLOMe treatment. Colocalization of GFP-TrafE (green) with Alexa Fluor $64710 \mathrm{kDa}$ dextran (red) $10 \mathrm{~min}$ after LLOMe treatment. Images were taken every $5 \mathrm{~min}, 5 \mathrm{~min}$ before $(\mathrm{t}=0)$ and $55 \mathrm{~min}$ after LLOMe treatment. Scale bars correspond to 10 $\mu \mathrm{m}$.

Movie S3. TrafE is required for optimal mebrane damage response. WT, atgl $\mathrm{KO}$ or trafE KO cells were stained with propidium iodide (PI) and monitored by live confocal time-lapse microscopy every $1 \mathrm{~min}, 5 \mathrm{~min}$ before and $55 \mathrm{~min}$ after addition of $5 \mathrm{mM}$ LLOMe. No LLOMe control to assess the effect of the fluorescent excitation on the cell fitness. 
Fig 1

A

D

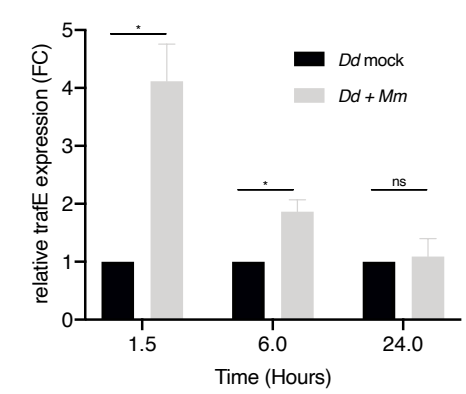

3 hpi

(1).
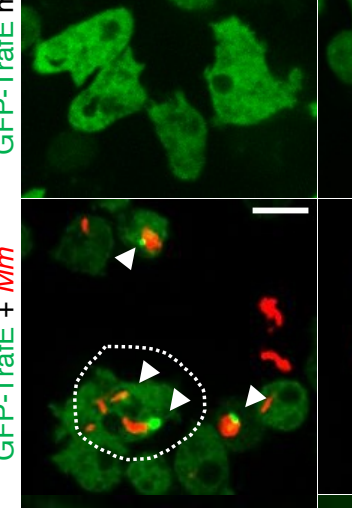
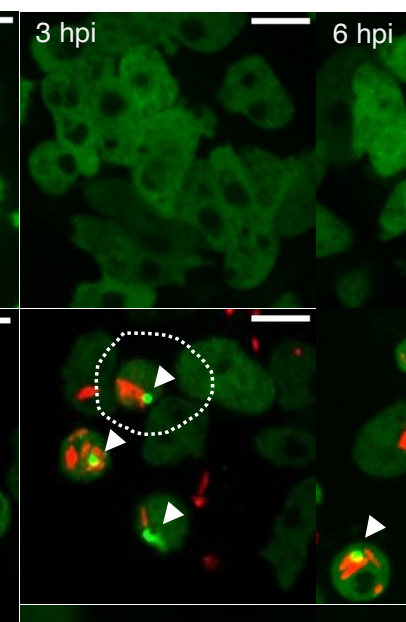

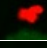

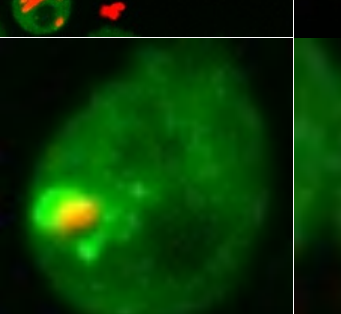

E

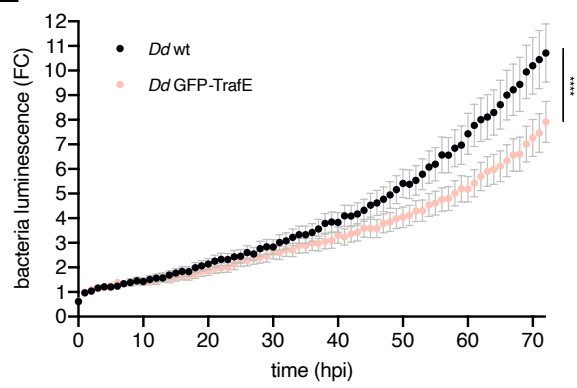

B

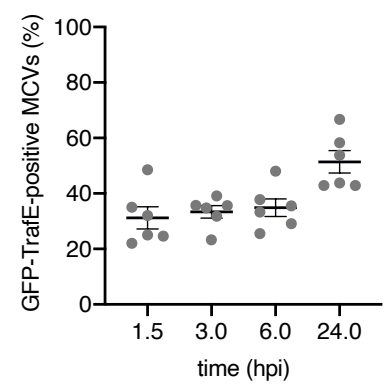

C

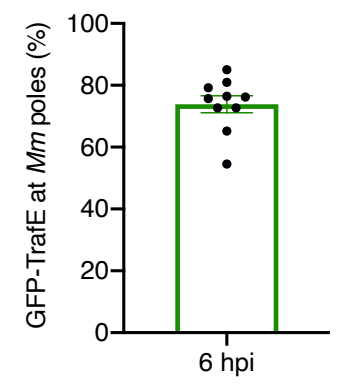

F

TrafE-GFP. KI + Mm

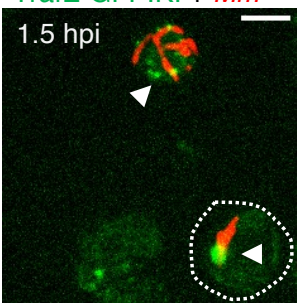

zoom

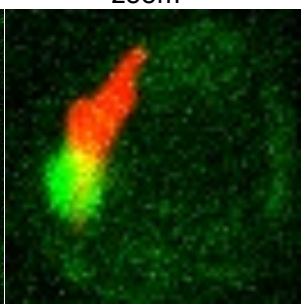


Fig 2

A

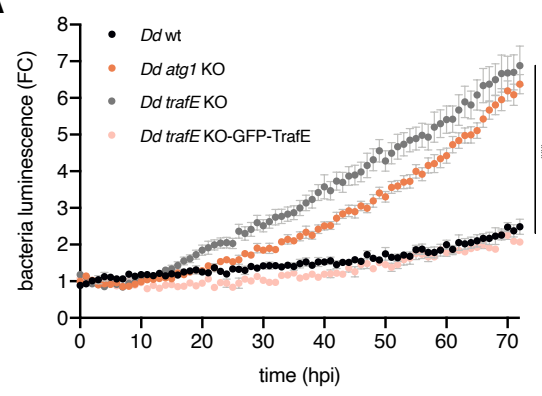

C

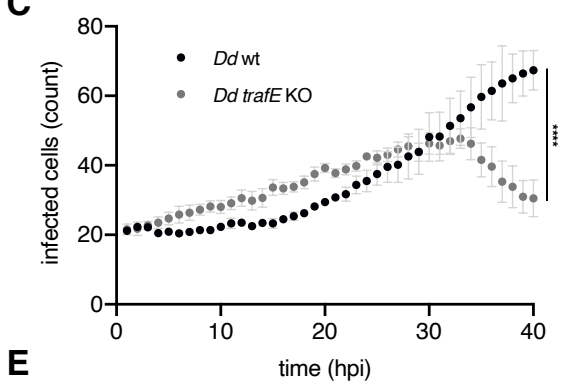

B
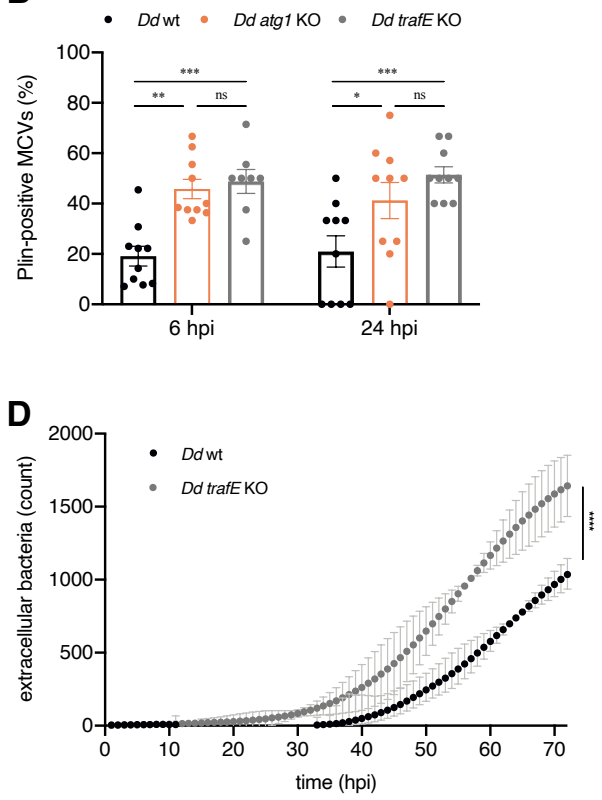

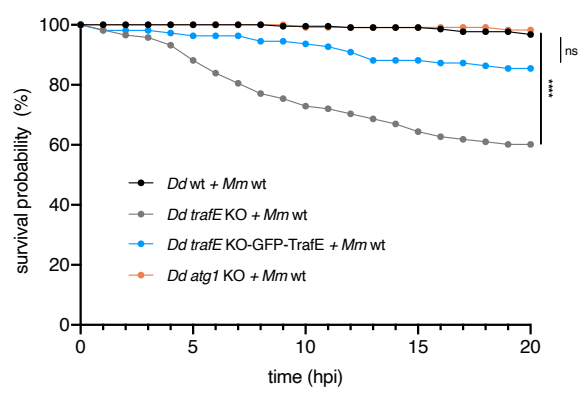


Fig 3

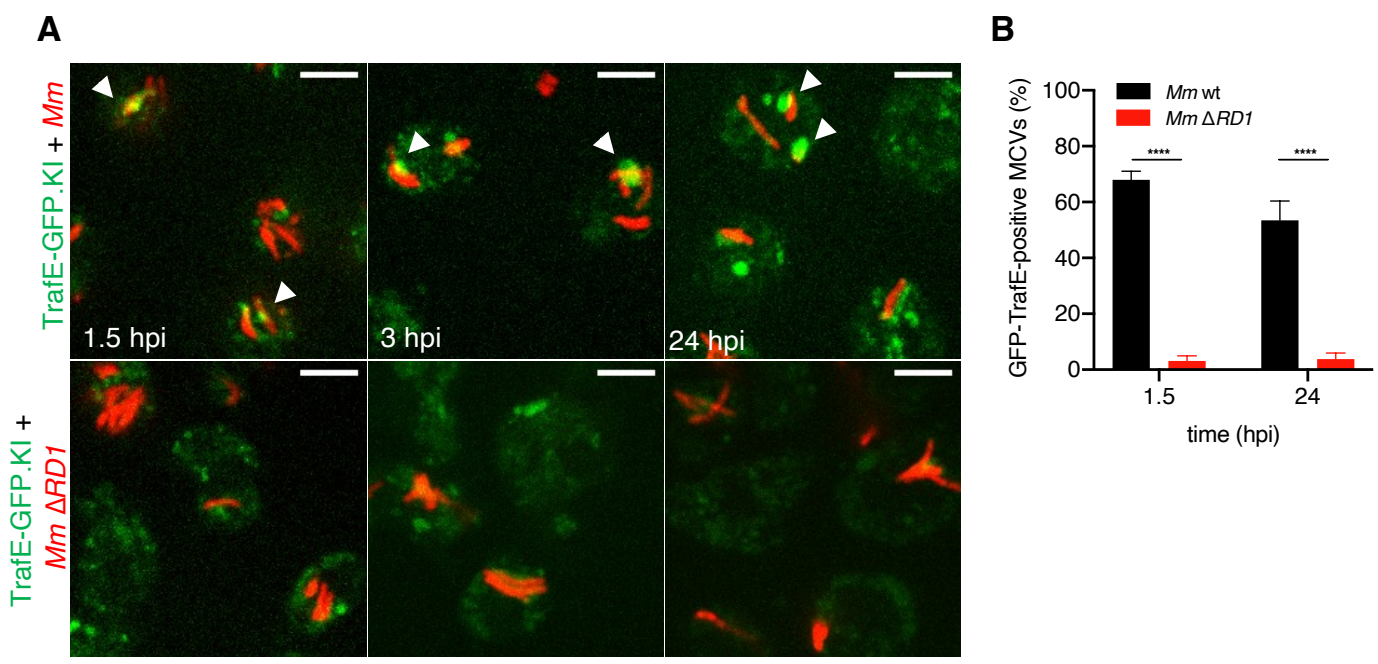

C

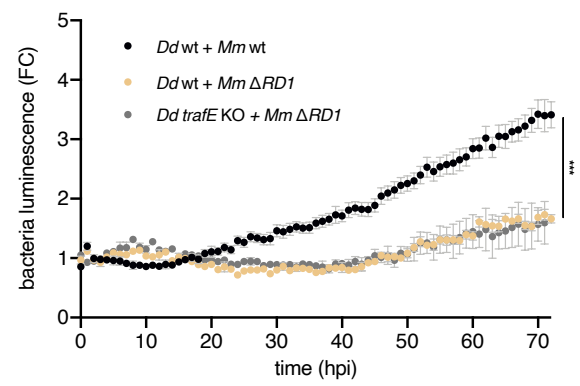

D

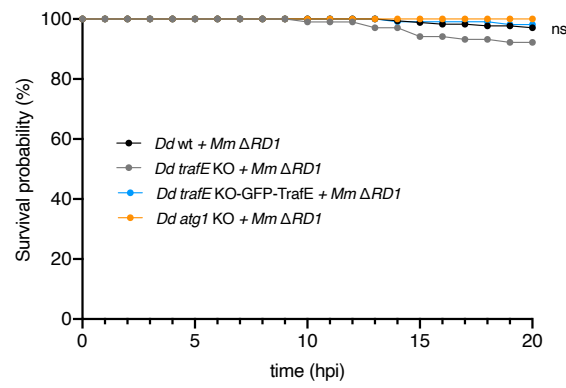


Fig 4
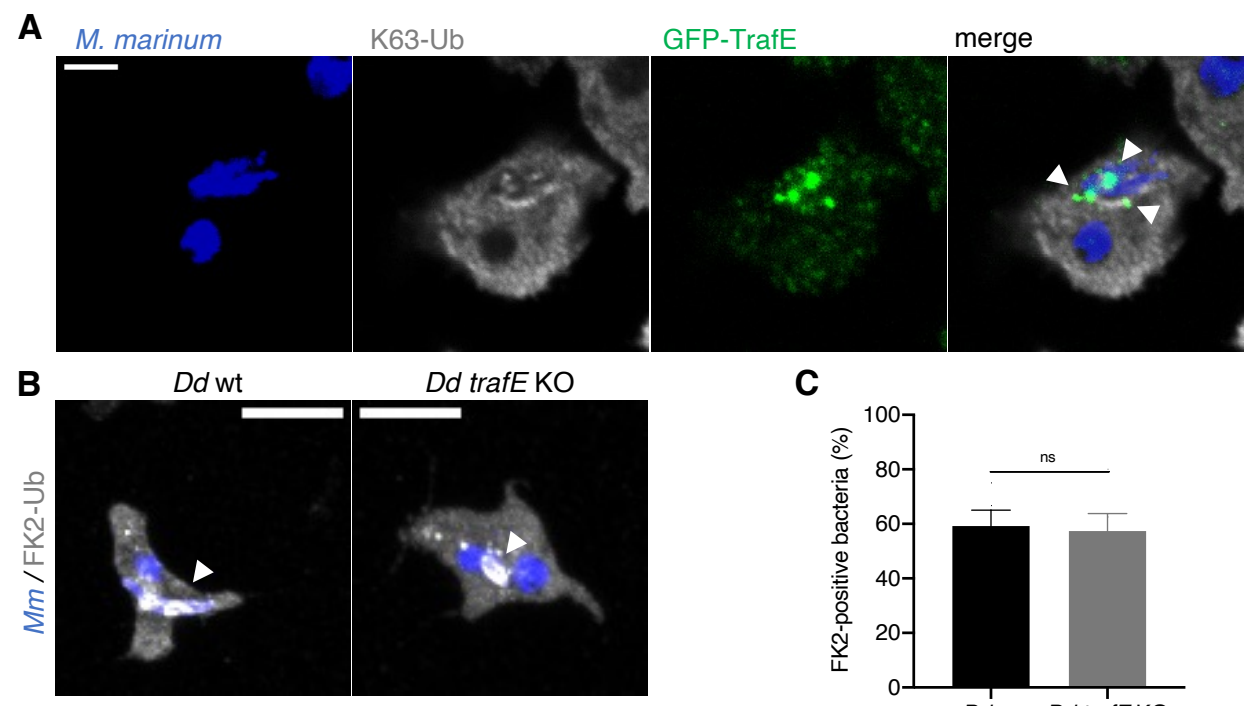

C
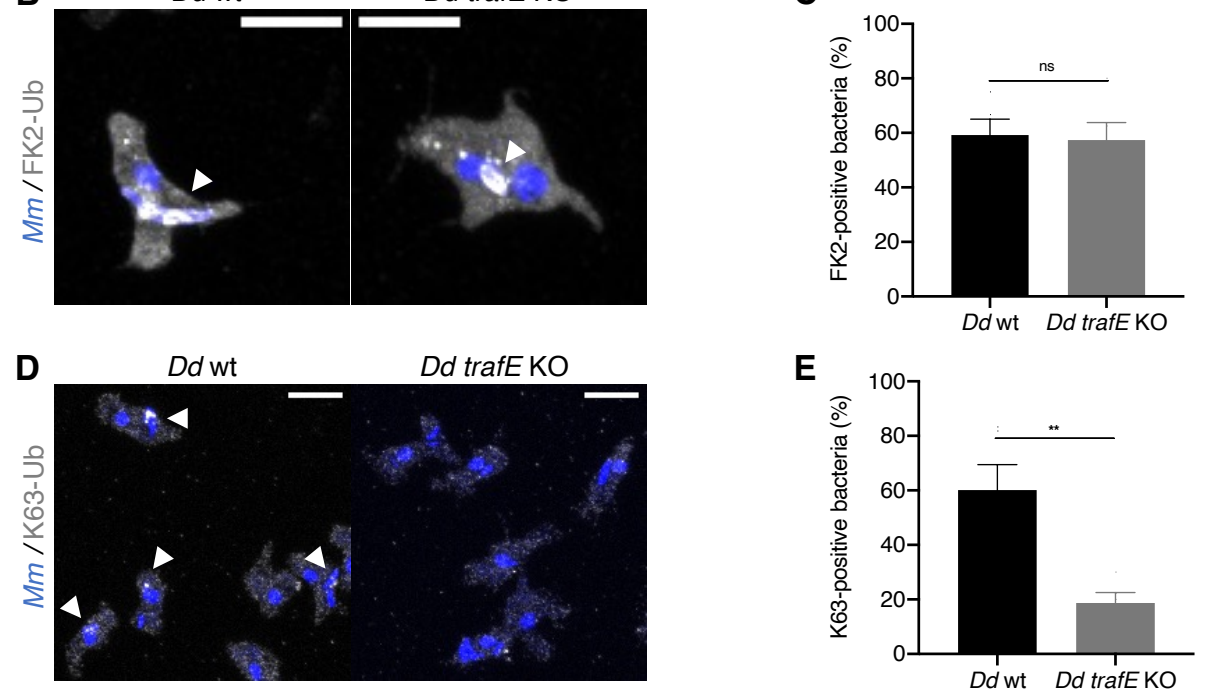

Fig 5

A

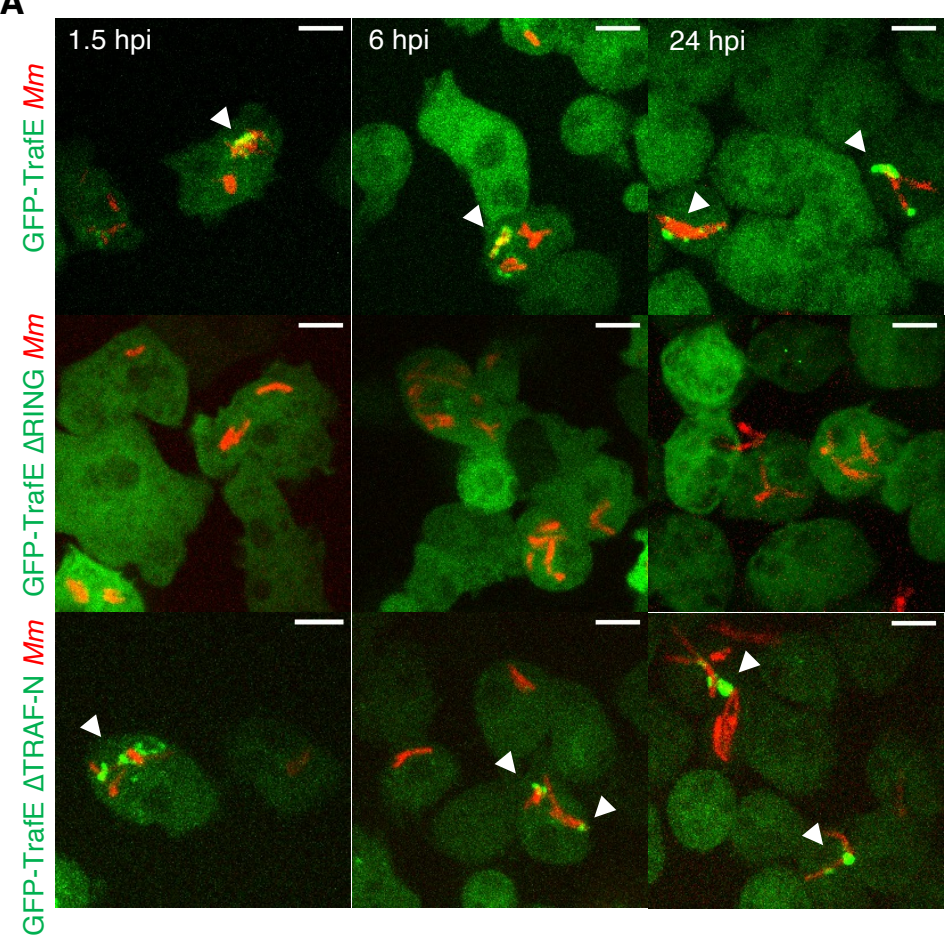

B

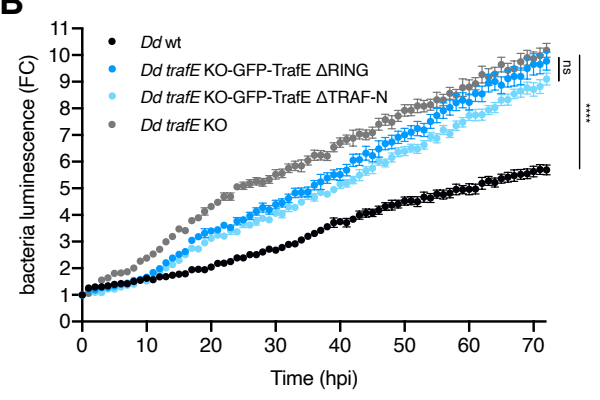


Fig 6

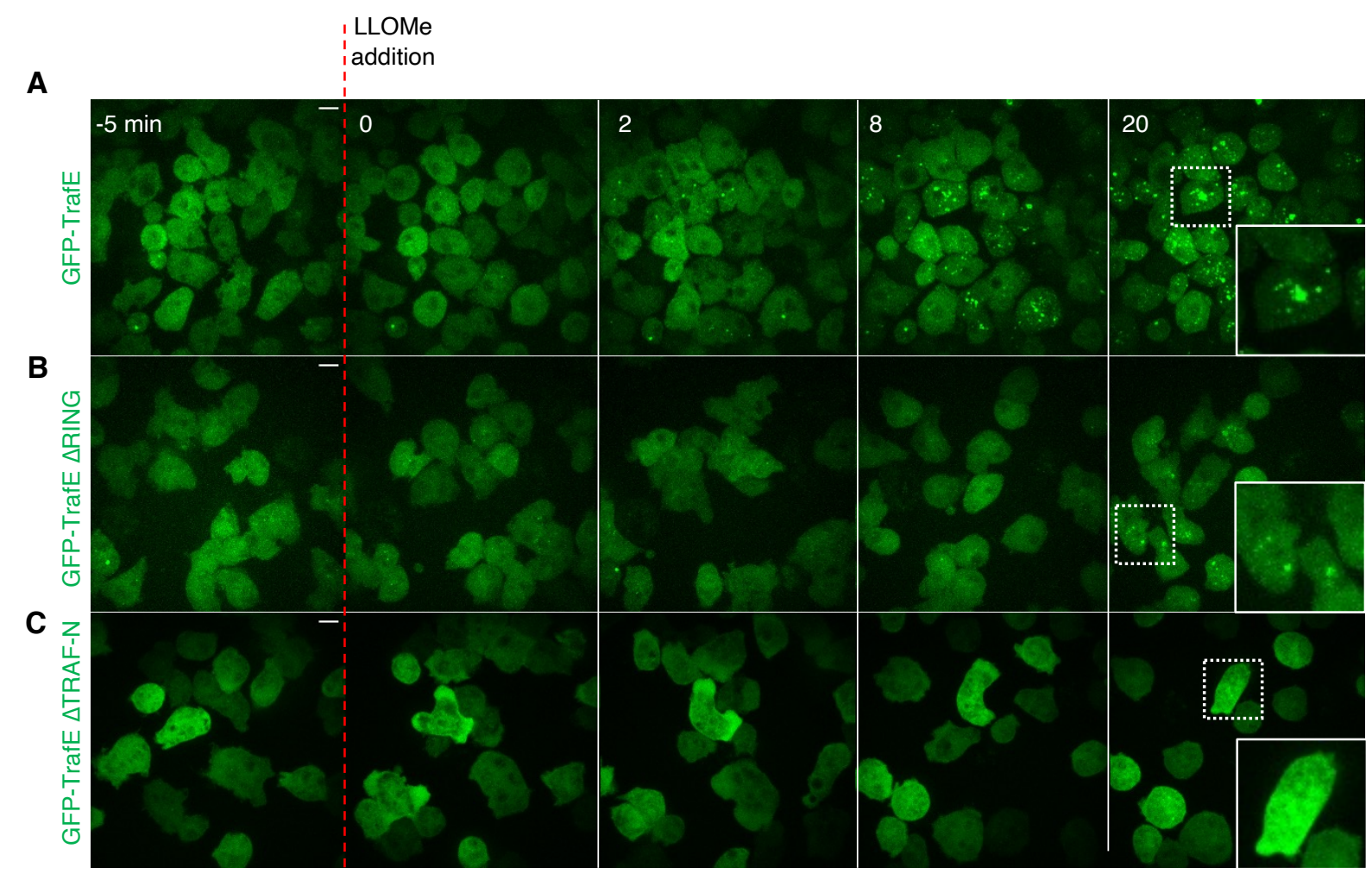

D

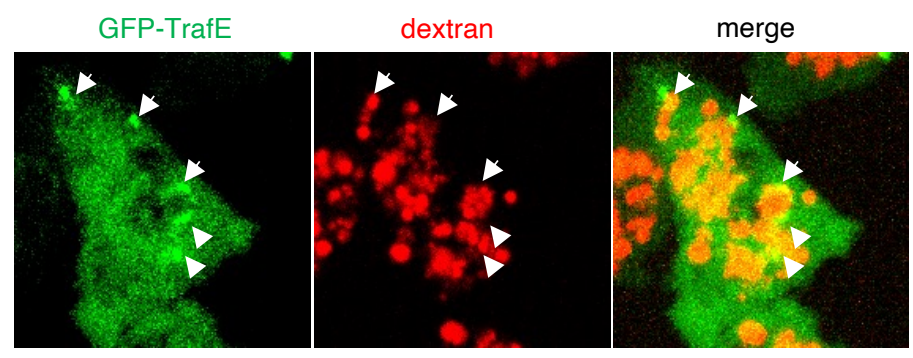

Fig 7

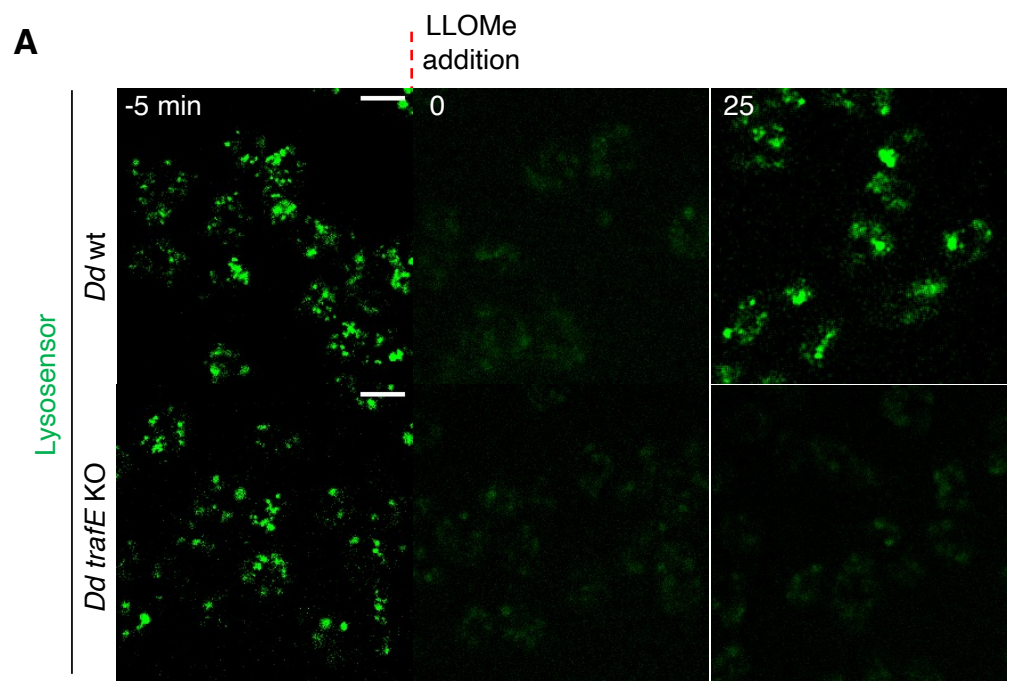

B

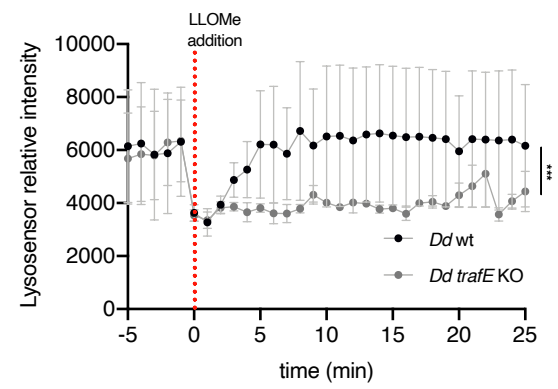


Fig 8

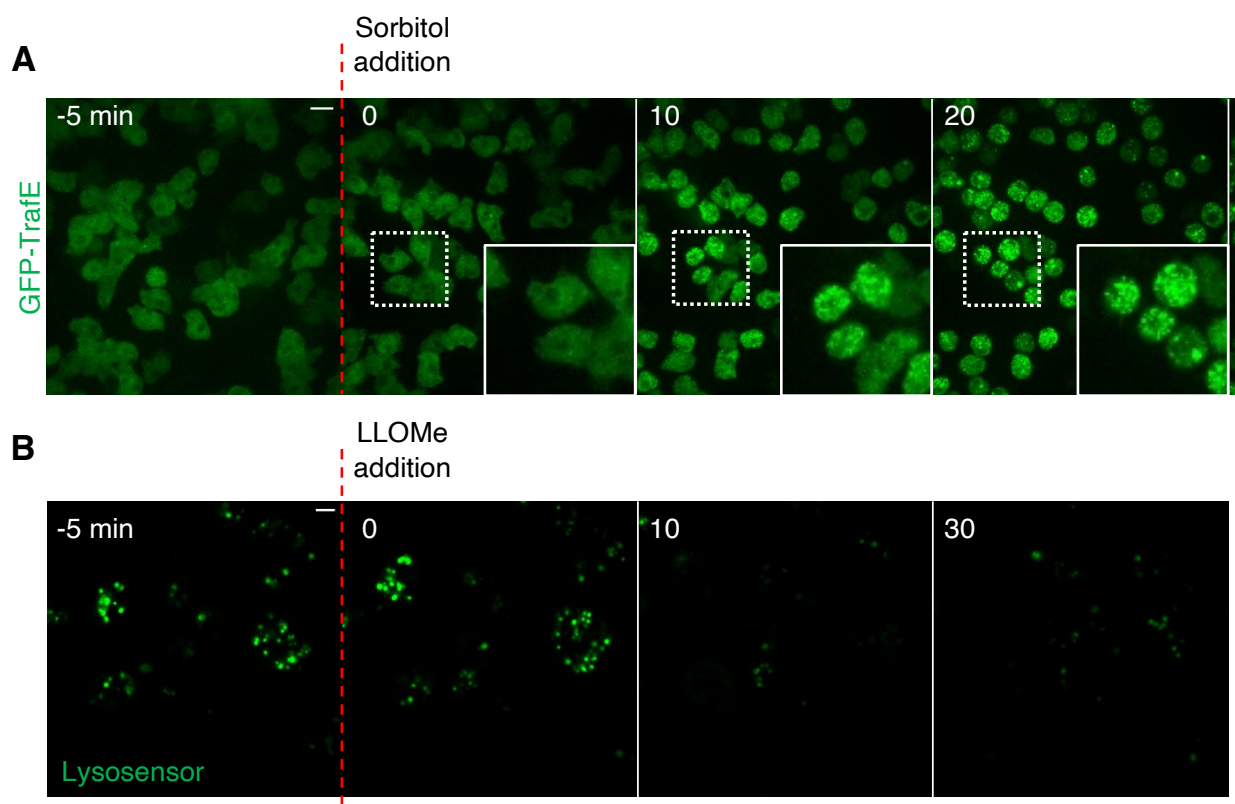

Sorbitol

30

55

addition

C
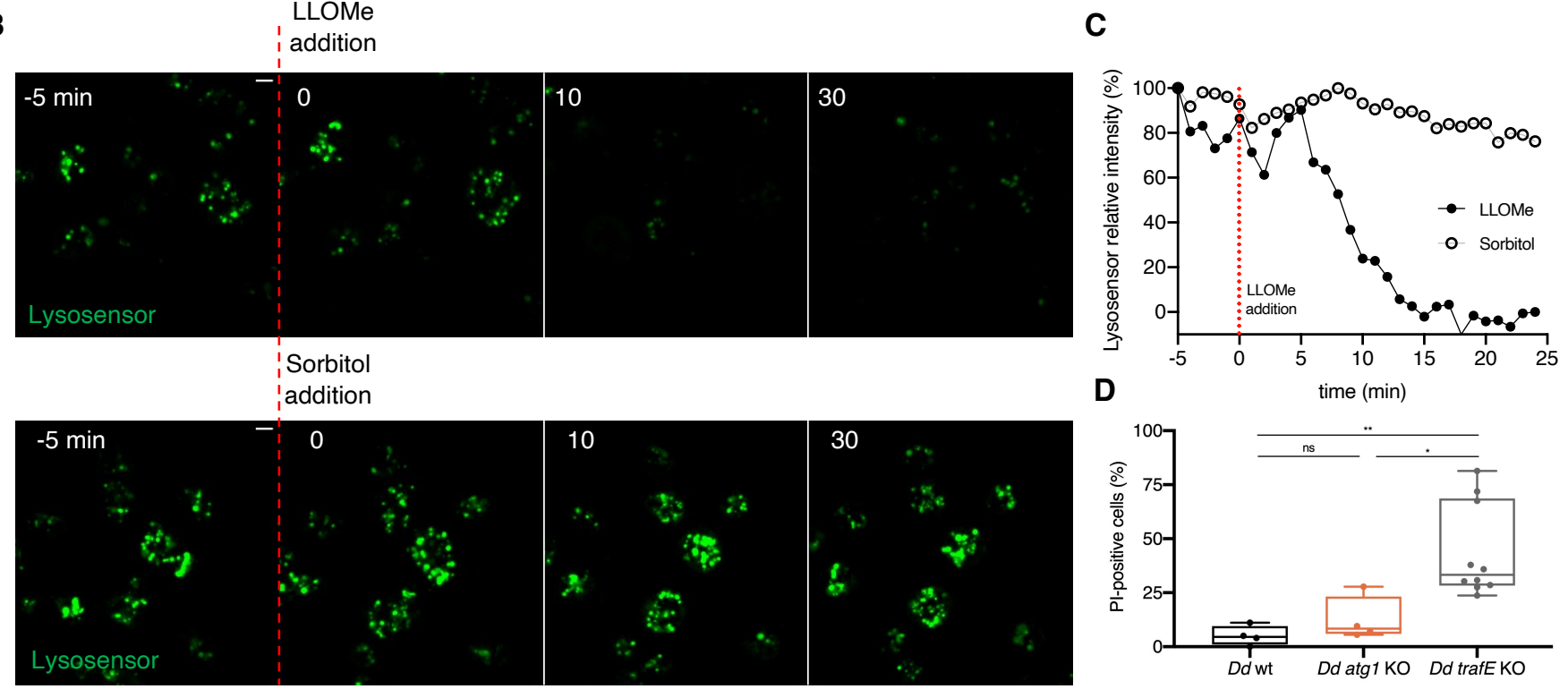


\section{Fig 9}

A

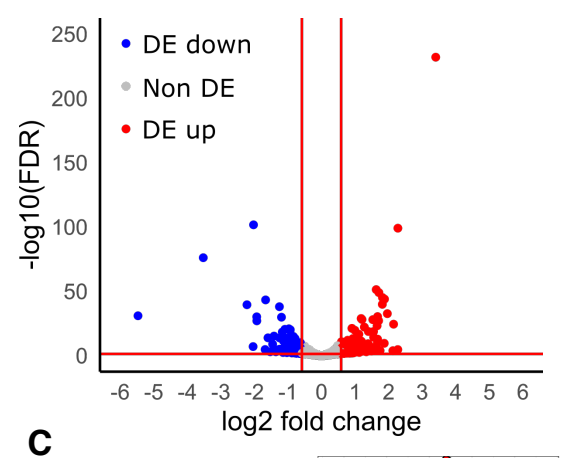

B
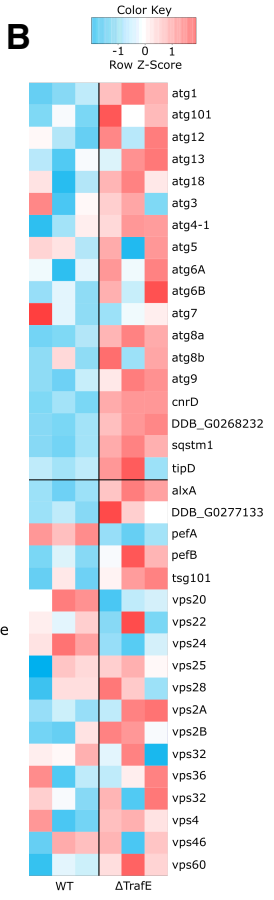

Fig 10

A

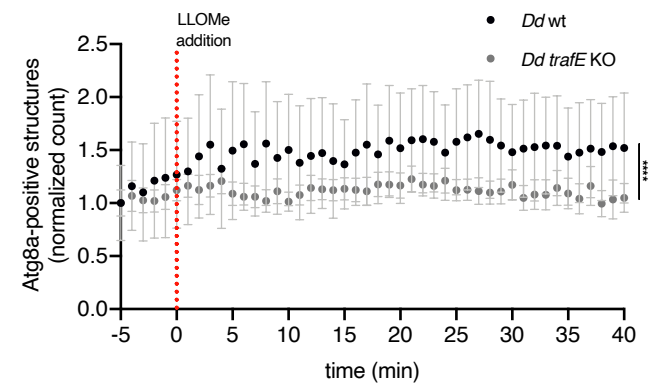

C

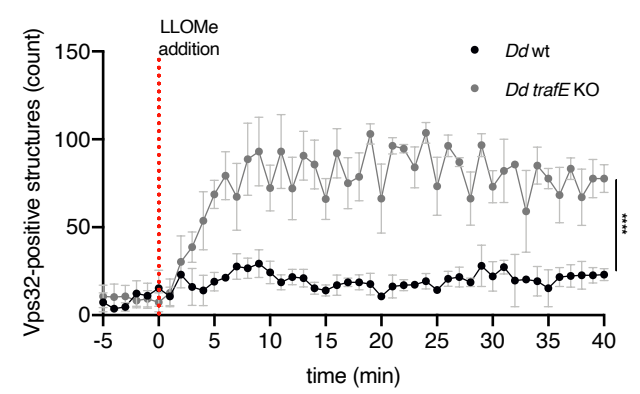

B

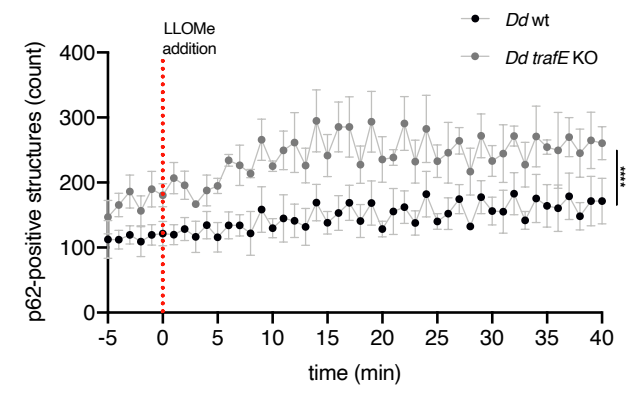

D

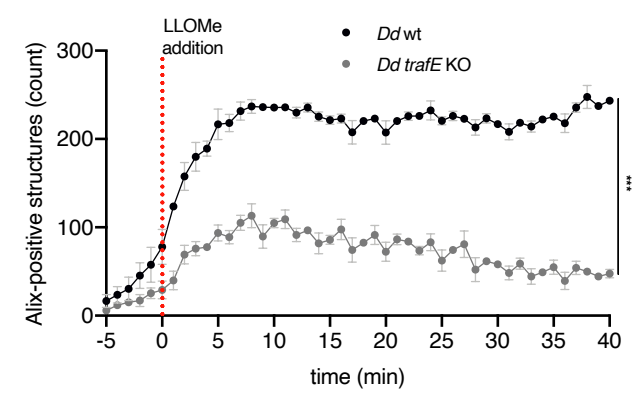

